\title{
Multiscale brain MRI super-resolution using deep 3D convolutional networks
}

\author{
Chi-Hieu Pham ${ }^{\mathrm{a}, *}$, Carlos Tor-Díez ${ }^{\mathrm{a}}$, Hélène Meunier ${ }^{\mathrm{b}}$, Nathalie Bednarek ${ }^{\mathrm{b}, \mathrm{d}}$, Ronan \\ Fablet $^{\mathrm{c}}$, Nicolas Passat $^{\mathrm{d}}$, François Rousseau $^{\mathrm{a}}$ \\ ${ }^{a}$ IMT Atlantique, LaTIM U1101 INSERM, UBL, Brest, France \\ ${ }^{b}$ Service de médecine néonatale et réanimation pédiatrique, $C H U$ de Reims, France \\ ${ }^{c} I M T$ Atlantique, LabSTICC UMR CNRS 6285, UBL, Brest, France \\ ${ }^{d}$ Université de Reims Champagne-Ardenne, CReSTIC, Reims, France
}

\begin{abstract}
The purpose of super-resolution approaches is to overcome the hardware limitations and the clinical requirements of imaging procedures by reconstructing high-resolution images from low-resolution acquisitions using post-processing methods. Super-resolution techniques could have strong impacts on structural magnetic resonance imaging when focusing on cortical surface or fine-scale structure analysis for instance. In this paper, we study deep three-dimensional convolutional neural networks for the super-resolution of brain magnetic resonance imaging data. First, our work delves into the relevance of several factors in the performance of the purely convolutional neural network-based techniques for the monomodal super-resolution: optimization methods, weight initialization, network depth, residual learning, filter size in convolution layers, number of the filters, training patch size and number of training subjects. Second, our study also highlights that one single network can efficiently handle multiple arbitrary scaling factors based on a multiscale training approach. Third, we further extend our super-resolution networks to the multimodal super-resolution using intermodality priors. Fourth, we investigate the impact of transfer learning skills onto super-resolution performance in terms of generalization among different datasets. Lastly, the learnt models are used to enhance real clinical low-resolution images. Results tend to demonstrate the potential of deep neural networks with respect to practical medical image applications.
\end{abstract}

Keywords: super-resolution, 3D convolutional neural network, brain MRI

\footnotetext{
*Corresponding author

Email addresses: ch.pham@imt-atlantique.fr (Chi-Hieu Pham), carlos.tordiez@imt-atlantique.fr (Carlos Tor-Díez), hmeunier@chu-reims.fr (Hélène Meunier), nbednarek@chu-reims.fr (Nathalie Bednarek), ronan.fablet@imt-atlantique.fr (Ronan Fablet), nicolas.passat@univ-reims.fr (Nicolas Passat), francois.rousseau@imt-atlantique.fr (François Rousseau)
} 


\section{Introduction}

Magnetic Resonance Imaging (MRI) is a powerful imaging modality for in vivo brain visualization with a typical image resolution of $1 \mathrm{~mm}$. Acquisition time of MRI data and signal-to-noise ratio are two parameters that drive the choice of an appropriate image resolution for a given study. The accuracy of further analysis such as brain morphometry can be highly dependent on image resolution. Super-Resolution (SR) aims to enhance the resolution of an imaging system using single or multiple data acquisitions (Milanfar 2010). Increasing image resolution through super-resolution is a key to more accurate understanding of the anatomy (Greenspan 2008). Previous works have shown that applying super-resolution techniques leads to more accurate segmentation maps of brain MRI data (Rueda et al. 2013; Tor-Díez et al. 2019) or cardiac data (Oktay et al. 2016).

The use of SR techniques has been studied in many works in the context of brain MRI analysis: structural MRI (Manjón et al. 2010b; Rousseau et al. 2010a; Manjón et al. 2010a; Rueda et al. 2013; Shi et al. 2015), diffusion MRI (Scherrer et al. 2012; Poot et al. 2013; Fogtmann et al. 2014; Steenkiste et al. 2016), spectroscopy MRI (Jain et al. 2017), quantitative $T_{1}$ mapping (Ramos-Llordén et al. 2017; Van Steenkiste et al. 2017), fusion of orthogonal scans of moving subjects (Gholipour et al. 2010; Rousseau et al. 2010b; Kainz et al. 2015; Jia et al. 2017). The development of efficient and accurate SR techniques for 3D MRI data could be a major step forward for brain studies.

Most SR methods rely on the minimization of a cost function consisting of a fidelity term related to an image acquisition model and a regularization term that constrains the space of solutions. The observation model is usually a linear model including blurring, motion, the effect of the point spread function (PSF) and downsampling. The regularizer, which guides the optimization process while avoiding unwanted image solutions, can be defined using pixel-based $\ell_{2}$-norm term (Gholipour et al. 2010), total variation (Shi et al. 2015), local patch-based similarities (Manjón et al. 2010b,a; Rousseau et al. 2010a), sparse coding (Rueda et al. 2013), low rank property (Shi et al. 2015).

In particular, the choice of this regularization term remains difficult as it modifies implicitly the space of acceptable solutions without any guaranty on the reconstruction of realistic high-resolution images. Conversely, in a supervised context (in which one can exploit a learning database with low-resolution (LR) and high-resolution (HR) images), the SR of MRI data can be fully driven by examples. The key challenge of supervised techniques is then to accurately estimate the mapping operator from the LR image space to the HR one. Recently, significant advances were reported in SR for computer vision using convolutional neural networks (CNN). This trend follows the tremendous outcome of CNN-based schemes for a wide range of computer vision applications, including for instance image classification (Krizhevsky et al. 2012; Simonyan and Zisserman 2014; He et al. 2016), medical image segmentation (Kamnitsas et al. 2017) or medical image analysis (Tajbakhsh et al. 2016).

CNN architectures have become the state-of-the-art for image SR. Initially, Dong et al. (2016a) proposed a three-layer CNN architecture. The first convolutional layer implicitly extracts a set of feature maps for the input LR image; the second layer maps these feature maps nonlinearly to HR patch representations; and the third layer reconstruct the HR 
image from these patch representations. Several studies have further investigated CNNbased architectures for image SR. Among others, the following features have been reported to improve SR performance: an increased depth of the network (Kim et al. 2016a), residual block (with batch normalization and skip connection) (Ledig et al. 2017), sub-pixel layer (Shi et al. 2016), perceptual loss function (instead of mean squared error-based cost functions) (Johnson et al. 2016; Ledig et al. 2017; Zhao et al. 2017), recurrent networks (Kim et al. 2016b), generative adversarial networks (Ledig et al. 2017; Pham et al. 2019). Very recently, Chen et al. (2018) proposed a 3D version of densely connected networks (Huang et al. 2017) for brain MRI SR. Inspired by the work by Jog et al. (2016), Zhao et al. (2018) investigated self super-resolution for MRI using enhanced deep residual networks (Lim et al. 2017).

Recently, very deep architectures obtained the best performance in a challenge focusing on natural image SR (NTIRE 2017 challenge, Timofte et al. 2017). However, due to the variety of the proposed methods and the high number of parameters for the networks architecture design, it is currently difficult to identify the key elements of a CNN architecture to achieve good performance for image SR and assess their applicability in the context of 3D brain MRI. In addition the extension of CNN architectures to 3D images, taking into account floating and possibly anisotropic scaling factors may be of interest to address the wide range of possible clinical acquisition settings, whereas classical CNN architectures only address a predefined (integer) scaling factor. The availability of multimodal imaging setting also questions the ability of CNN architectures to benefit from such multimodal data to improve the SR of a given modality.

Contributions: This work presents a comprehensive review of deep convolutional neural networks, and associated key elements, for brain MRI SR. Following Timofte et al. (2016), who have experimentally shown several ways to improve SR techniques from a baseline architecture, we study the impact of eight key elements on the performance of convolutional neural networks for 3D brain MRI SR. We demonstrate empirically that residual learning associated with appropriate optimization methods can significantly reduce the time of the training step and fast convergence can be achieved in 3D SR context. Overall, we report better performance when learning deeper fully $3 \mathrm{D}$ convolution neural networks and using larger filters. Interestingly, we demonstrate that a single network can handle multiple arbitrary scale factors efficiently, for example, from $2 \times 2 \times 2 \mathrm{~mm}$ to $2 \times 2 \times 1 \mathrm{~mm}$ or $1 \times 1 \times 1 \mathrm{~mm}$, by learning multiscale residuals from spline-interpolated image. We also report significant improvement using a multimodal architecture, where a HR reference image can guide the CNN-based SR of a given MRI volume. Moreover, we demonstrate that our model can transfer the rich information available from high-resolution experimental dataset to lower-quality clinical image data.

\section{Super-Resolution using Deep Convolutional Neural Networks}

\subsection{Learning-based $S R$}

Single image SR is a typically ill-posed inverse problem that can be stated according to the following linear formulation:

$$
\mathbf{Y}=\mathbf{H X}+\mathbf{N}=D_{\downarrow} B \mathbf{X}+\mathbf{N}
$$


where $\mathbf{Y} \in \mathbb{R}^{n}$ and $\mathbf{X} \in \mathbb{R}^{m}$ denote a LR image and a HR image, $\mathbf{H} \in \mathbb{R}^{m \times n}$ is the observation matrix $(m>n)$ and $\mathbf{N}$ denotes an additive noise. $D_{\downarrow}$ represents the downsampling operator and $B$ is the blur matrix. The purpose of SR methods is to estimate $\mathbf{X}$ from the observations $\mathbf{Y}$. The SR image can be estimated by minimizing a least-square cost function:

$$
\widehat{\mathbf{X}}=\arg \min _{\mathbf{X}}\|\mathbf{Y}-\mathbf{H X}\|^{2} .
$$

The minimization of the Equation (2) usually leads to unstable solutions and requires the use of appropriate regularization terms on $\mathbf{X}$. Adding prior knowledge on the image solution (such as piecewise smooth image) may lead to unrealistic solution. In a learning-based context where a set of image pairs $\left(\mathbf{X}_{i}, \mathbf{Y}_{i}\right)$ is available, the objective is to learn the mapping from the LR images $\mathbf{Y}_{i}$ to the HR images $\mathbf{X}_{i}$, leading to the following formulation:

$$
\widehat{\mathbf{X}}=\arg \min _{\mathbf{X}}\left\|\mathbf{X}-\mathbf{H}^{-1} \mathbf{Y}\right\|^{2} .
$$

In this setting, the matrix $\mathbf{H}^{-1}$ can be modeled as a combination of a restoration matrix $F \in \mathbb{R}^{m \times m}$ and an upscaling interpolation operator $S^{\uparrow}: \mathbb{R}^{n} \rightarrow \mathbb{R}^{m}$. Given a set of $\mathrm{HR}$ images $\mathbf{X}_{i}$ and their corresponding LR images $\mathbf{Y}_{i}$ with $K$ samples, the restoration operator $F$ can be estimated as follows:

$$
\widehat{F}=\arg \min _{F} \sum_{i=1}^{K}\left\|\mathbf{X}_{i}-F\left(S^{\uparrow} \mathbf{Y}_{i}\right)\right\|^{2}=\arg \min _{F} \sum_{i=1}^{K}\left\|\mathbf{X}_{i}-F\left(\mathbf{Z}_{i}\right)\right\|^{2}
$$

where $\mathbf{Z} \in \mathbb{R}^{m}$ is the interpolated LR (ILR) version of $\mathbf{Y}$ (i.e. $\mathbf{Z}=S^{\uparrow} \mathbf{Y}$ ). $\quad F$ is then a mapping from the ILR image space to the HR image space.

$\mathrm{SR}$ is the process of estimating HR data from LR data. The main goal is then to estimate high-frequency components from LR observations. Instead of learning the mapping directly from the LR space to the HR one, it might be easier to estimate a mapping from the LR space to the missing high-frequency components, also called the residual between $\mathrm{HR}$ and LR data: $\mathbf{R}=\mathbf{X}-\mathbf{Z}$ or equivalently $\mathbf{X}=\mathbf{Z}+\mathbf{R}$. This approach can be modeled by a skip connection in the network. In such a residual-based modeling, one typically assumes that $\mathbf{R}$ is a function of $\mathbf{Z}$. The computation of HR data is then expressed as follows: $\mathbf{X}=\mathbf{Z}+F(\mathbf{Z})$ where $F$ can be learnt using the following equation:

$$
\widehat{F}=\arg \min _{F} \sum_{i=1}^{K}\left\|\left(\mathbf{X}_{i}-\mathbf{Z}_{i}\right)-F\left(\mathbf{Z}_{i}\right)\right\|^{2} .
$$

\subsection{CNN-based baseline architecture}

In this paper, we focus on the learning of the mapping $F$ with convolutional neural networks. Following Dong et al. (2016a) and Kim et al. (2016a), the mapping $F$ from $\mathbf{Z}$ to $(\mathbf{X}-\mathbf{Z})$ is decomposed into nonlinear operations that correspond to the combination of convolution-based and rectified linear unit (ReLU) layers.

The baseline architecture used in this work can be described as follows: 


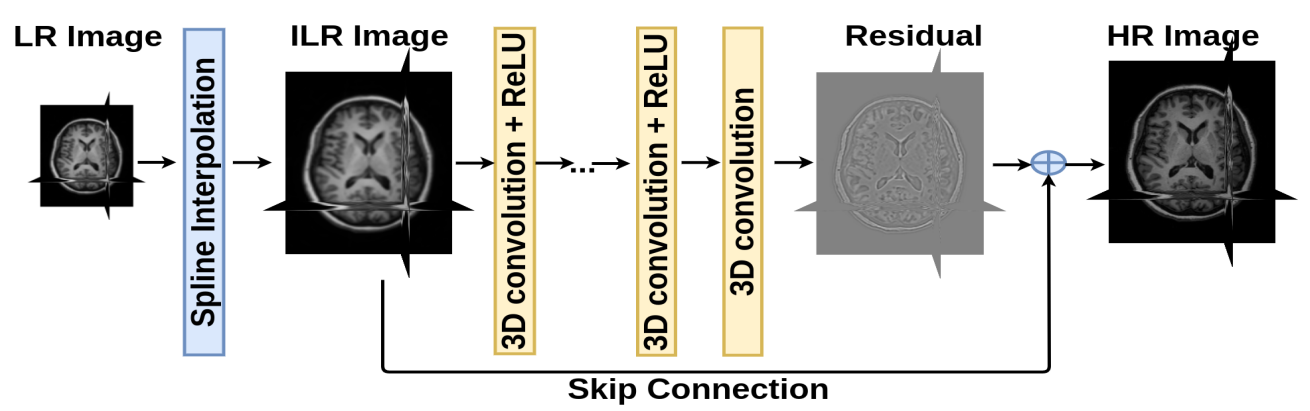

Figure 1: 3D deep neural network for single brain MRI super-resolution.

$$
\left\{\begin{array}{c}
F_{1}(\mathbf{Z})=\max \left(0, W_{1} * \mathbf{Z}+B_{1}\right) \\
F_{i}(\mathbf{Z})=\max \left(0, W_{i} * F_{i-1}(\mathbf{Z})+B_{i}\right) \text { for } 1<i<L \\
F_{L}(\mathbf{Z})=W_{L} * F_{L-1}(\mathbf{Z})+B_{L}
\end{array}\right.
$$

where:

- $L$ is the number of layers,

- $W_{i}$ and $B_{i}$ are the parameters of convolution layers to learn. $W_{i}$ corresponds to $n_{i}$ convolution filters of support $c \times f_{i} \times f_{i} \times f_{i}$, where $c$ is the number of channels in the input of layer $i, f_{i}$ and $n_{i}$ are respectively the spatial size of the filters and the number of filters of layer $i$,

- $\max (0, \cdot)$ refers to a ReLU applied to the filter responses.

This network architecture is depicted in Figure 1. Please note that, for instance, the SRCNN model proposed by Dong et al. 2016a corresponds to a specific parameterization of this baseline architecture with $f_{1}=9, f_{2}=1, f_{3}=5, n_{1}=64, n_{2}=32$ and no skip connection.

The performance of a given architecture depends on several parameters such as the filter size $f_{i}$, the number of filters $n_{i}$, the number of layers $L$, etc. Understanding how these parameters influence the reconstruction of the HR image with respect to the considered application setting (e.g., number of training samples, image size, scaling factor) is a key issue, which remains poorly explored. For instance, regarding the number of layers, it is commonly believed that the deeper the better (Simonyan and Zisserman 2014; Kim et al. 2016a). However, adding layers increases the number of parameters and can lead to overfitting. In particular, previous works (Dong et al. 2016a; Oktay et al. 2016), have shown that a deeper structure does not always lead to better results (Dong et al. 2016a).

Specifically focusing on MRI data, the specific objectives of this study are: i) the evaluation and understanding of the effect of key elements of CNN for brain MRI SR, ii) the experimental study of arbitrary multiscale SR using CNN, iii) investigating multimodalityguided SR using CNN. 


\section{Sensitivity Analysis of the Considered Architecture}

In this section, we present the MRI datasets used for evaluation and the key elements of CNN architecture to achieve good performance for single image SR.

\subsection{MRI Datasets and LR simulation}

To evaluate SR performances of CNN-based architectures, we have used two MRI datasets: the Kirby 21 dataset and the NAMIC Brain Multimodality dataset.

The Kirby 21 dataset (Landman et al. 2011) consists of MRI scans of twenty-one healthy volunteers with no history of neurological conditions. Magnetization prepared gradient echo (MPRAGE, T1-weighted) scans were acquired using a 3T MR scanner (Achieva, Philips Healthcare, The Netherlands) with a $1.0 \times 1.0 \times 1.2 \mathrm{~mm}^{3}$ resolution over an FOV of $240 \times$ $204 \times 256 \mathrm{~mm}$ acquired in the sagittal plane. Flair data were acquired using $1.1 \times 1.1 \times 1.1 \mathrm{~mm}^{3}$ resolution over an FOV of $242 \times 180 \times 200 \mathrm{~mm}$ acquired in the sagittal plane. The T2-weighted volumes were acquired using a 3D multi-shot turbo-spin echo (TSE) with a TSE factor of 100 with over an FOV of $200 \times 242 \times 180 \mathrm{~mm}$ including a sagittal slice thickness of $1 \mathrm{~mm}$.

MR images of NAMIC Brain Multimodality ${ }^{1}$ dataset have been acquired using a $3 \mathrm{~T}$ GE at BWH in Boston, MA. An 8 Channel coil was used in order to perform parallel imaging using ASSET (Array Spatial Sensitivity Encoding techniques, GE) with a SENSEfactor (speed-up) of 2. The structural MRI acquisition protocol included two MRI pulse sequences. The first results in contiguous spoiled gradient-recalled acquisition (fastSPGR) with the following parameters: $\mathrm{TR}=7.4 \mathrm{~ms}, \mathrm{TE}=3 \mathrm{~ms}, \mathrm{TI}=600,10$ degree flip angle, $25.6 \mathrm{~cm}^{2}$ field of view, matrix $=256 \times 256$. The voxel dimensions are $1 \times 1 \times 1 \mathrm{~mm}^{3}$. The second XETA (eXtended Echo Train Acquisition) produces a series of contiguous T2-weighted images $\left(\mathrm{TR}=2500 \mathrm{~ms}, \mathrm{TE}=80 \mathrm{~ms}, 25.6 \mathrm{~cm}^{2}\right.$ field of view, $1 \mathrm{~mm}$ slice thickness $)$. Voxel dimensions are $1 \times 1 \times 1 \mathrm{~mm}^{3}$.

As in Shi et al. 2015 and Rueda et al. 2013, LR images have been generated from a Gaussian blur and a down-sampling by isotropic scaling factors. In the training phase, a set of patches of training images is randomly extracted. In the baseline setting, the training dataset comprises 10 subjects (3200 patches $25 \times 25 \times 25$ per subject randomly sampled) and the testing dataset is composed of 5 subjects. During the testing step, the network is applied on the whole images. The peak signal-to-noise ratio (PSNR) in decibels $(\mathrm{dB})$ is used to evaluate the SR results with respect to the original HR images. No denoising or bias correction algorithms were applied to the data. Image intensity has been normalized between 0 and 1 . The following figures are drawn based on the average PSNR over all test images.

\subsection{Baseline and benchmarked architectures}

The network architecture that is used as a baseline approach in this study is illustrated in Figure 1. The baseline network is a 10 blocks (convolution+ReLU) network with the following parameters: 64 convolution filters of size $(3 \times 3 \times 3)$ at each layer, mean squared

\footnotetext{
${ }^{1}$ NAMIC : http://hdl.handle.net/1926/1687
} 
error (MSE) as loss function, weight initialization by He et al. (2015) (MSRA filler), Adam (adaptive moment estimation) method for optimization (Kingma and Ba 2015), 20 epochs on Nvidia GPU and using Caffe package (Jia et al. 2014), batch size of 64, learning rate set to 0.0001 , no regularization or drop out has been used. The learning rate multipliers of weights and biases are 1 and 0.1, respectively. For benchmarking purposes, we consider two other state-of-the-art SR models: low-rank total variation (LRTV) (Shi et al. 2015) and SRCNN3D (Pham et al. 2017). SRCNN3D (Pham et al. 2017), which is a 3D extension of the method described in (Dong et al. 2016a), has 3 convolutional layers with the size of $9^{3}$, $1^{3}$ and $5^{3}$, respectively. The layers of SRCNN3D consist of 64 filters, 32 filters and one filter, respectively.

The next sections present the impact of the key parameters studied in this work: optimization method, weight initialization, residual-based model, network depth, filter size, filter number, training patch size and size of training dataset.

\subsection{Optimization Method}

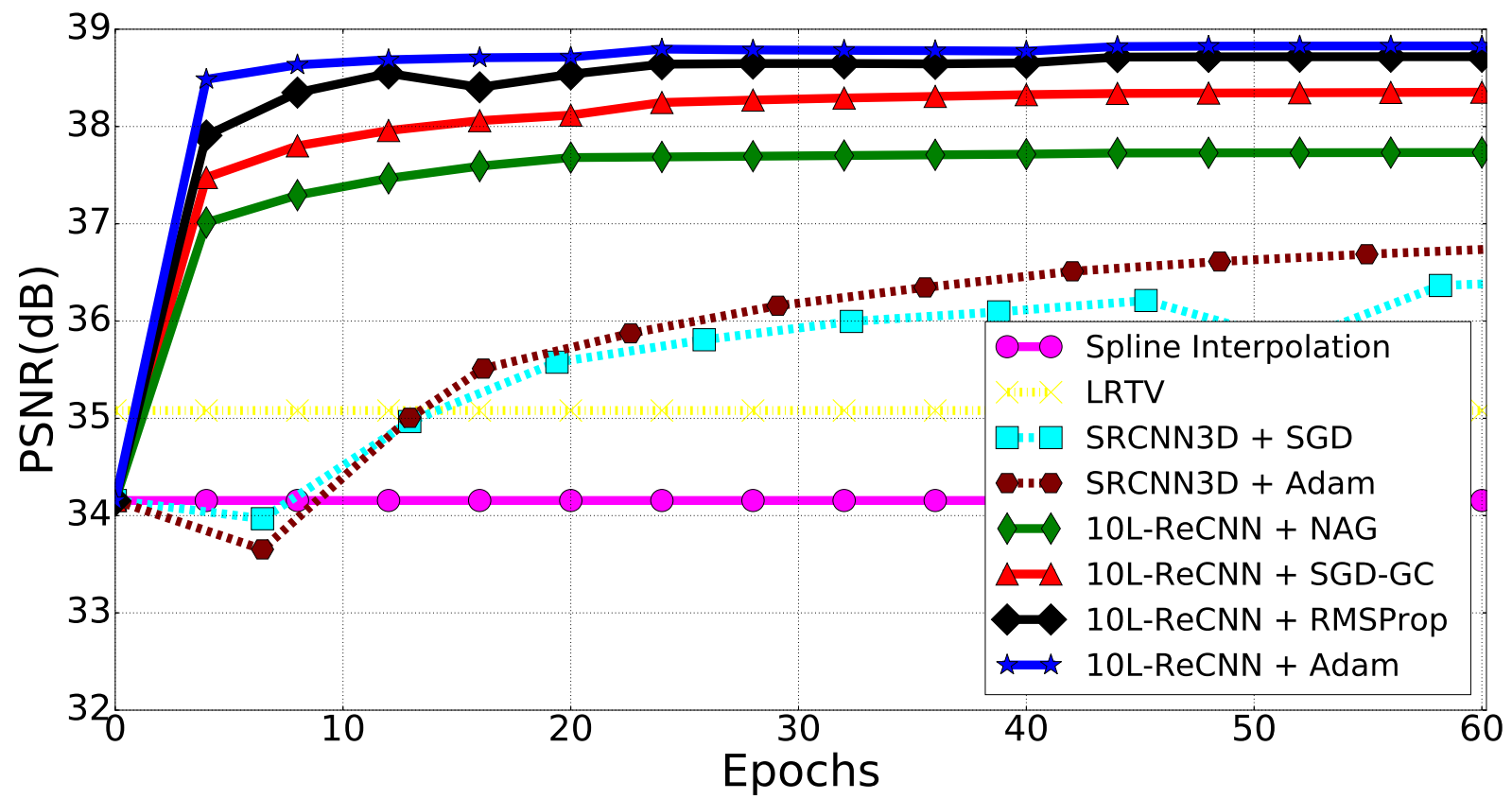

Figure 2: Impact of the optimization methods onto SR performance: SGD-GC, NAG, RMSProp and Adam optimisation of a 10L-ReCNN (10-layer residual-learning network with $f=3$ and $n=64$ ). We used Kirby 21 for training and testing with isotropic scaling factor $\times 2$. The initial learning rates of SGD-GC, NAG, RMSProp and Adam are set respectively to $0.1,0.0001,0.0001$ and 0.0001 . These learning rates are decreased by a factor of 10 every 20 epochs. The momentum of these methods, except RMSProp, is set to 0.9. All optimization methods use the same weight initialization described by He et al. (2015).

Given a training dataset which consists of pairs of LR and HR images, network parameters are estimated by minimizing the objective function using optimization algorithms. These algorithms play a very important role in training neural networks. The more efficient and 
effective optimization strategies lead to faster convergence and better performance. More precisely, during the training step, the estimation of the restoration operator $F$ corresponds to the minimization of the objective function $\mathcal{L}$ in Equation (5) over network parameters $\boldsymbol{\theta}=\left\{W_{i}, B_{i}\right\}_{i=1, \ldots, L}$

Most optimization methods for CNNs are based on gradient descent. A classical method applies a mini-batch stochastic gradient descent with momentum (SGD) (LeCun et al. 1998) as used by Dong et al. (2016a); Pham et al. (2017). However, the use of fixed momentum causes numerical instabilities around the minimum. Nesterov's accelerated gradient (NAG) (Nesterov 1983) was proposed to cope with this issue but the use of small learning rates induces slow convergence. By contrast, high learning rates may lead to exploding gradients (Bengio et al. 1994; Glorot and Bengio 2010). In order to address this issue, Kim et al. (2016a) proposed the stochastic gradient descent method with an adjustable gradient clipping (SGD-GC) (Pascanu et al. 2013) to achieve an optimization with high learning rates. The predefined range over which gradient clipping is applied may still cause SGD-GC not to converge quickly or make difficult the tuning of a global learning rate. Recently, methods have been proposed to address this issue through an automatic adaption of the learning rate for each parameter to be learnt. RMSProp (root-mean-square propagation) (Tieleman and Hinton 2012) and Adam (adaptive moment estimation) (Kingma and Ba 2015) are the two most popular models in this category.

The results of four optimization methods (NAG, SGD-GC, RMSProp and Adam) for the baseline network are illustrated in Figure 2. Firstly, regardless the method used, the baseline network shows better performance than LRTV (Shi et al. 2015) and SRCNN3D (Pham et al. 2017). Secondly, it can be observed that the baseline network can converge very rapidly (only 20 epochs with small learning rate of 0.0001 ). Finally, in these experiments, the most efficient and effective optimization method is Adam as regards both PSNR metric and convergence speed. Hence, in the next sections, we use Adam method with $\beta_{1}=0.9$ and $\beta_{2}=0.999$ to train our networks with 20 epochs.

\subsection{Weight Initialization}

The optimization algorithms for training a CNN are typically initialized randomly. Inappropriate initialization can lead to long time convergence or even divergence. Several studies (Dong et al. 2016a; Oktay et al. 2016; Pham et al. 2017) used a normal distribution $\mathcal{N}(0,0.001)$ to initialize the weights of convolutional filters. However, because of too small initial weights, the optimizer may be stuck into a local minimum especially when building deeper networks. Both Dong et al. (2016a) concluded that deeper networks do not lead to better performance, and Oktay et al. (2016) confirmed that the addition of extra convolutional layers to the 7-layer model is found to be ineffective. Uniform distribution $\mathcal{U}\left(-\sqrt{3 /\left(n f^{3}\right)}, \sqrt{3 /\left(n f^{3}\right)}\right)$ (called Xavier filler) (Glorot and Bengio 2010) was also proposed to initialize the weights of deeper networks. In order to add more layers to networks, He et al. (2015) suggested an initial training stage by sampling from the normal distribution $\mathcal{N}\left(0, \sqrt{2 /\left(n f^{3}\right)}\right)$ (called here Microsoft Research Asia - MSRA filler).

Overall, we evaluate here the weight initialization schemes described by Glorot and Bengio (2010) and He et al. (2015), a normal distribution $\mathcal{N}(0,0.001)$ as proposed by Dong et al. 


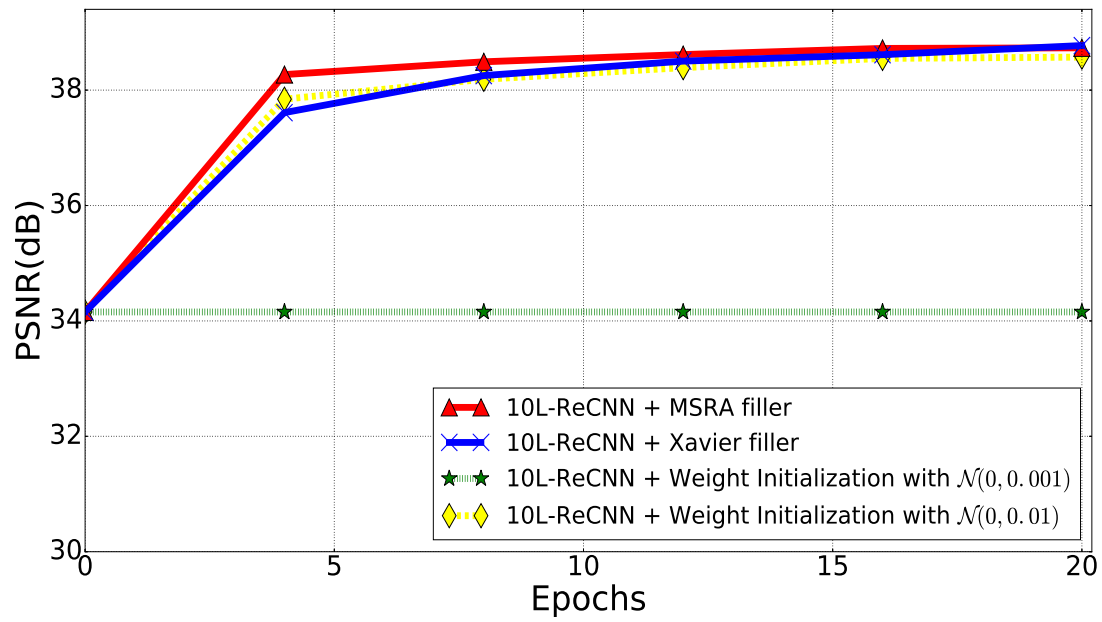

(a) : 10-layer residual-learning networks (10L-ReCNN)

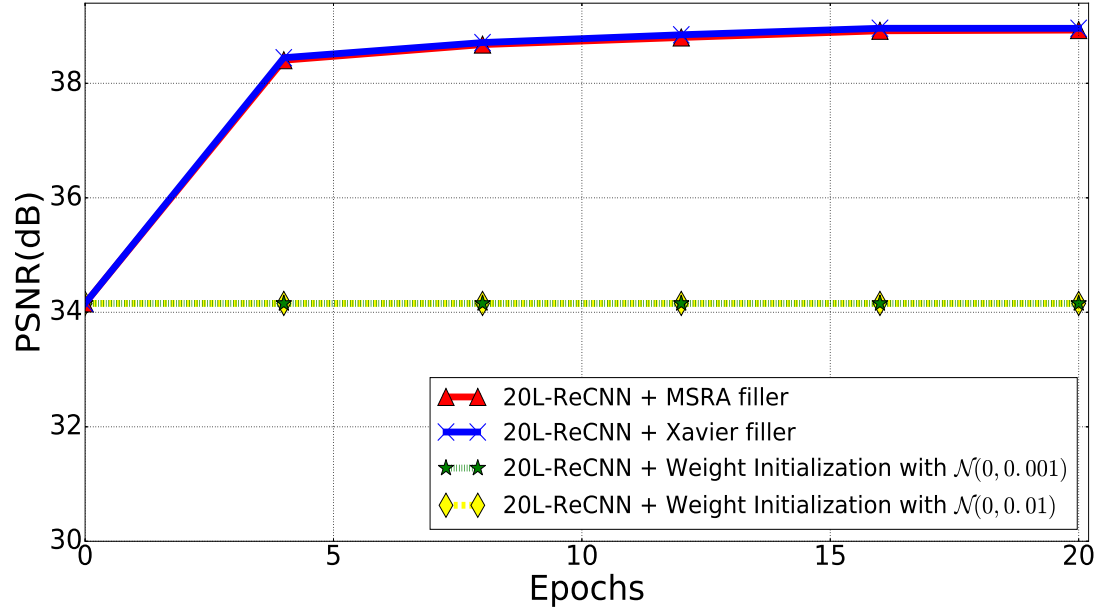

(b) : 20-layer residual-learning networks (20L-ReCNN)

Figure 3: Weight initialization scheme vs. performance (residual-learning networks with the same filter numbers $n=64$ and filter size $f=3$ using Adam optimization and tested with isotropic scaling factor $\times 2$ using Kirby 21 for training and testing, 32000 patches with size $25^{3}$ for training).

(2016a); Oktay et al. (2016) and a normal distribution $\mathcal{N}(0,0.01)$ for the considered SR architecture. Experiments with a deeper architecture were also performed, more precisely for the considered experimental setup due to GPU memory setting. As shown in Figure 3, the initialization with normal distributions $\mathcal{N}(0,0.001)$ failed to make the training of both 10-layer and 20-layer residual-learning networks converge. In addition, our 20-layer network also does not converge when initialized with normal distributions $\mathcal{N}(0,0.01)$. By contrast, MSRA and Xavier filler schemes make the networks converge and reach similar reconstruction performance. For the rest of this paper, we use MSRA weight filler as initialization scheme. 


\subsection{Residual Learning}

The CNN methods proposed by Dong et al. (2016a); Shi et al. (2016); Dong et al. (2016b) use the LR image as input and outputs the HR one. We refer to such approach as a non-residual learning. Within these approaches, low-frequency features are propagated through the layers of networks, which may increase the representation of redundant features in each layer and in turn the computational efficiency of the training stage. By contrast, one may consider residual learning or normalized HR patch prediction as pointed out by several learning-based SR methods (Zeyde et al. 2012; Timofte et al. 2013, 2014; Kim et al. 2016a). When considering CNN methods, one may design a network which predicts the residual between the HR image and the output of the first transposed convolutional layer (Oktay et al. 2016). Using residual blocks, a CNN architecture may implicitly embed residual learning while still predicting the HR image (Ledig et al. 2017).

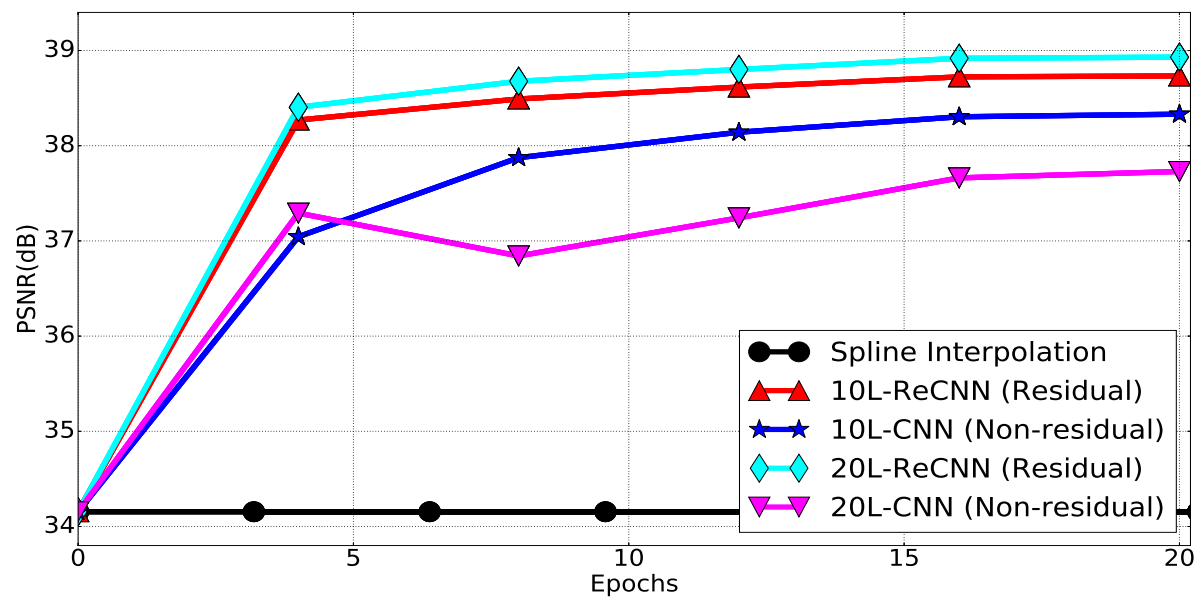

Figure 4: Non-residual-learning vs Residual-learning networks with the same $n=64$ and $f^{3}=3^{3}$ and the depths of 10 and 20 (called here 10L-CNN vs 10L-ReCNN and 20L-CNN vs 20L-ReCNN) over 20 training epochs using Adam optimization with the same training strategy and tested with isotropic scale factor $\times 2$ using Kirby 21 for training and testing.

Here, we perform a comparative evaluation of non-residual learning vs. residual learning strategies. Figure 4 depicts PSNR values and convergence speed of residual vs. non-residual network structures with 10 and 20 convolutional layers. The residual-learning networks converge faster than the non-residual-learning ones. In addition, residual learning leads to improvements in PSNR $(+0.4 \mathrm{~dB}$ for 10 layers and $+1.2 \mathrm{~dB}$ for 20 layers). It might be noted that these experiments do not support the common statement that the deeper, the better for CNNs. Here, the use of additional layers is only beneficial when using residual modeling. Deeper architectures may even lower the reconstruction performance with nonresidual learning.

\subsection{Depth, Filter Size and Number of Filters}

As shown by the previous experiment, the link between network depth and performance remains unclear. Besides, it is hard to train deeper networks because gradient computation 


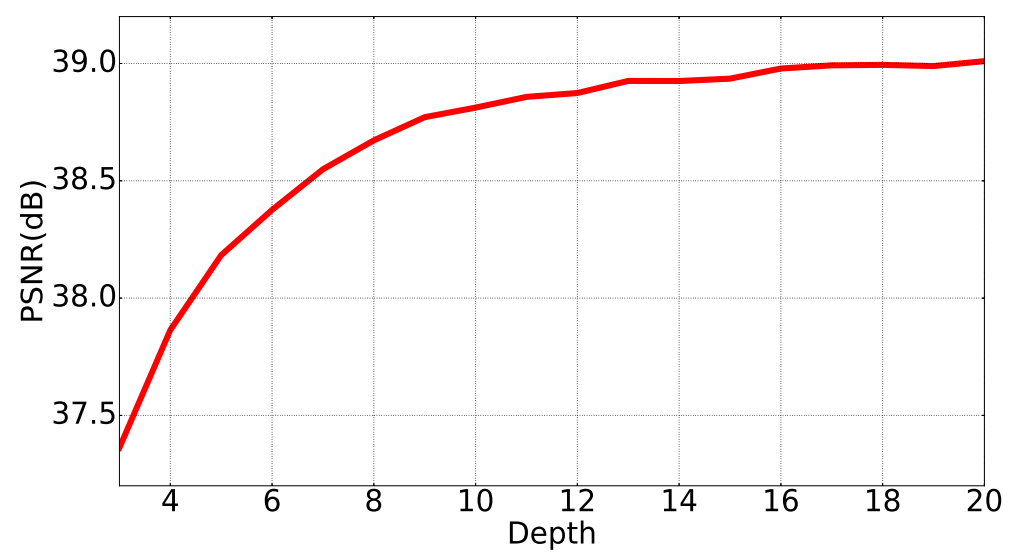

Figure 5: Depth vs Performance (residual-learning networks with the same filter numbers $n=64$ and filter size $f=3$ over 20 training epochs using Adam optimization and tested with isotropic scale factor $\times 2$ using Kirby 21 for training and testing, 32000 patches with size $25^{3}$ for training).

can be unstable when adding layers (Glorot and Bengio 2010). For instance, Oktay et al. (2016) tested extra convolutional layers to a 7-layer model but achieved negligible performance improvement. As mentioned in Section 2.2, SRCNN (Dong et al. 2016a) was also tested with deeper architectures but no improvement was reported. However, Kim et al. (2016a) argue that the performance of CNNs for SR could be improved by increasing the depth of network compared to neural network architectures proposed by Dong et al. (2016a); Oktay et al. (2016).

The previous section supports that deeper architectures may be beneficial when considering a residual learning. We now evaluate the reconstruction performance as a function of the number of layers. Results are reported in Figure 5. They stress that increasing network depth with residual learning improves the quality of the estimated HR image (e.g. $+1.6 \mathrm{~dB}$ increasing of the depth from 3 to 20 or $+0.5 \mathrm{~dB}$ increasing of the depth from 7 to 20 ).

The parameterization of the convolutional filters is also of key interest. Inspired by the VGG network designed for classification (Simonyan and Zisserman 2014), previous CNN methods for SR mostly focused on small convolutional filters of size $(3 \times 3 \times 3)$ as proposed by Kim et al. (2016a); Oktay et al. (2016); Kamnitsas et al. (2017). Oktay et al. (2016) even argued that such architecture can lead to better non-linear estimations. Regarding the number of filters for each layer, Dong et al. (2016a) reported greater reconstruction performance when increasing the number of filters. But these experiences were not reported in other CNN-based SR studies (Kim et al. 2016a; Oktay et al. 2016). Here, we both evaluate the effect of the filter size and of the number of filters.

Figure 6 shows that a 10-layer network with a filter size of $5^{3}$ shows results as well as a 20-layer network with $3^{3}$ filters. Besides reconstruction performance, the use of a larger filter size decreases the training speed and significantly increases the complexity and memory cost for training. For example, it took us 50 hours to train a 10-layer network with a filter size of $5^{3}$. By contrast, a deeper network with smaller filters (i.e. 20-layer network with $3^{3}$ filters) involves a smaller number of parameters, such that it took us only 24 hours to train. 

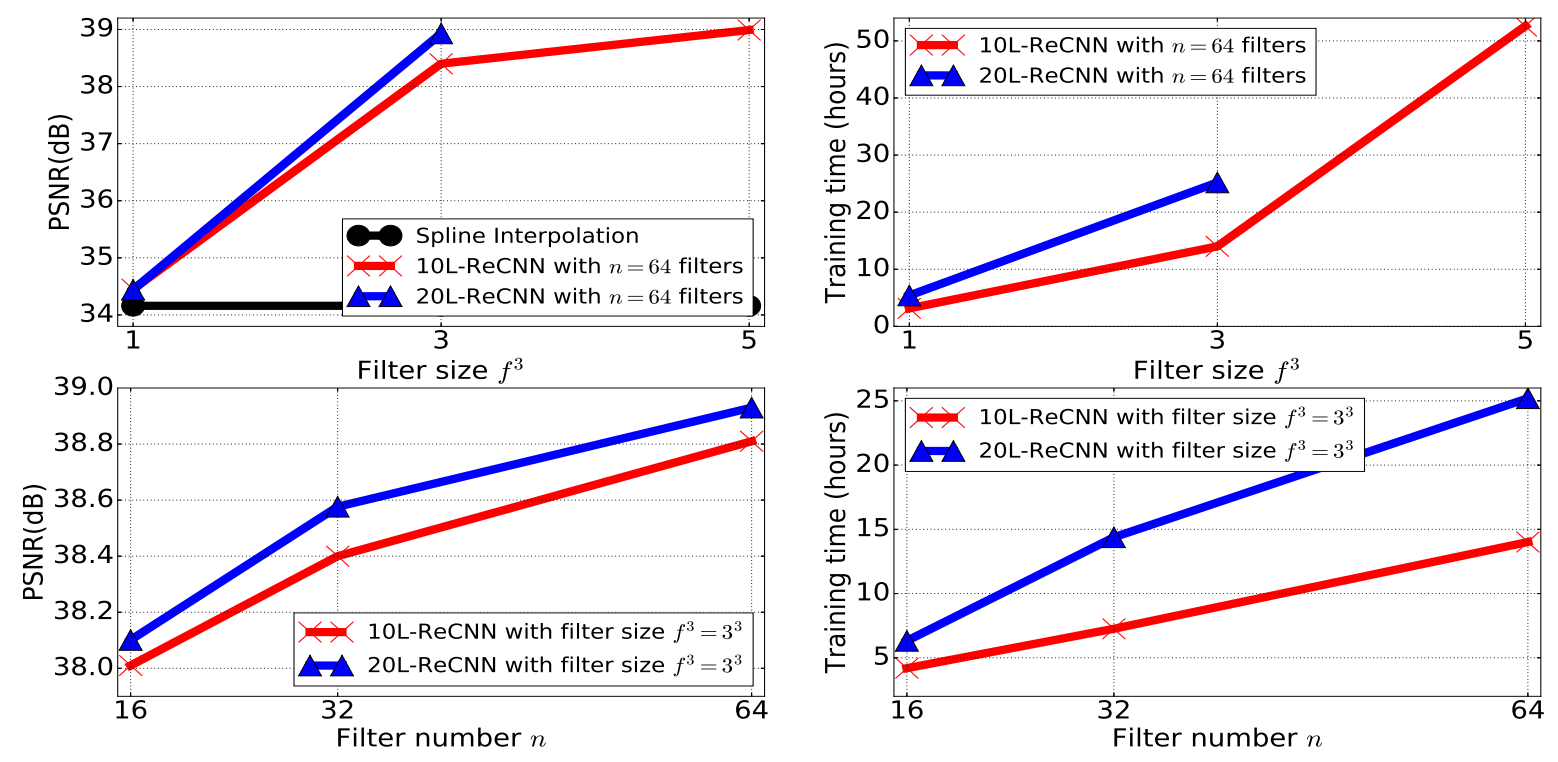

Figure 6: Impact of convolution filter parameters (sizes $f \times f \times f=f^{3}$ with $n$ filters) on PSNR and computation time. These 10-layers residual-learning networks are trained from scratch using Kirby 21 with Adam optimization over 20 epochs and tested with the testing images of the same dataset for isotropic scale factor $\times 2$.

These experiments suggest that deeper architectures with small filters can replace shallower networks with larger filters both in terms of computational complexity and of reconstruction performance. In addition, the increase in the number of filters within networks can increase the performance. However, we were not able to use 128 filters with the baseline architecture due to the limited amount of memory. This stresses out the need to design memory efficient architectures for 3D image processing using deeper CNNs with more filters.

\subsection{Training Patch Size and Subject Number}

In the context of brain MRI SR, the acquisition and collection of large datasets with homogeneous acquisition settings is a critical issue. We now evaluate the extent to which the number of training subjects influences SR reconstruction performance. As the training samples are extracted as patches of brain MRI images, we also evaluate the impact of the training patch size on learning and reconstruction performances.

The size of training patches should be greater or equal to the size of the receptive field of the considered network (Simonyan and Zisserman 2014; Kim et al. 2016a), which is given by $((f-1) D+1)^{3}$ for a $D$-layer network with filter size $f^{3}$. Figure 7 confirms that better performances can be achieved using larger training patches (from $11^{3}$ to $31^{3}$ with the 10-layer network and from $11^{3}$ to $29^{3}$ with the 12-layer network). However, if the patch size is larger than the receptive field (e.g. $21^{3}$ within the 10-layers network and $25^{3}$ within the 12-layers network), then the improvement is negligible consuming, in the meantime, considerably more GPU memory and training time.

We stressed previously that the selection of the network depth involves a trade-off between reconstruction performance and GPU memory requirement and training time increase. 

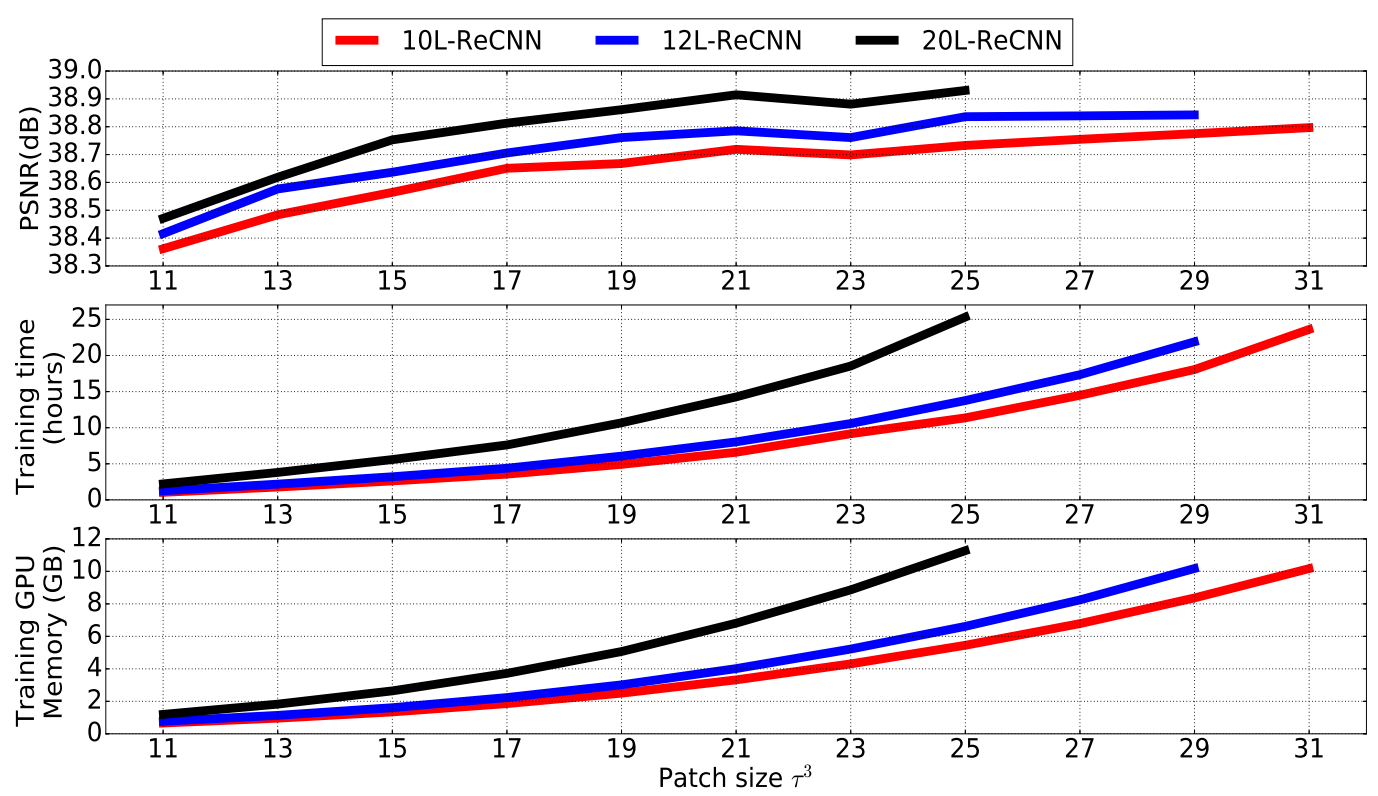

Figure 7: First row: Training patch size vs. performance. Second row: Patch size vs. training time. Third row: Patch size vs training GPU memory requirement. These networks with the same $n=64$ and $f^{3}=3^{3}$ are trained from scratch using Kirby 21 with batch of 64 and tested with the testing images of the same dataset for isotropic scale factor $\times 2$.

A similar result can be drawn with respect to the patch size. Figure 7 illustrates that larger training patch sizes also require more memory for training. Moreover, this figure shows that better performance can be achieved using larger training patch sizes. It may be noted that the performance of the 10-layer networks may reach a performance similar to 12-layer and 20-layer networks when using larger training patches but it takes more time and more GPU memory for training.

Regarding the number of training subjects, Figure 8 points out that a single subject is enough to reach better performance than spline interpolation. Interestingly, reconstruction performance increases slightly when more subjects are considered, which appears appropriate for real-world applications. However, in fact, more training dataset takes more time within the same experience settings. In the next sections, for saving training time, we propose to use 10 subjects for learning.

\section{Handling Arbitrary Scales}

In some CNN-based SR approaches, the networks are learnt for a fixed and specified scaling factor. Thus, a network built for one scaling factor cannot deal with any other scale. In medical imaging, Oktay et al. (2016) have applied CNNs for upscaling cardiac image slices with the scale of 5 (e.g. upscaling the voxel size from $1.25 \times 1.25 \times 10.0 \mathrm{~mm}$ to $1.25 \times 1.25 \times 2.00 \mathrm{~mm})$. Typically, their network is not capable of handling other scales due to the use of fixed deconvolutional layers. In brain MRI imaging, the variety of the possible acquisition settings motivates us to explore multiscale settings. 


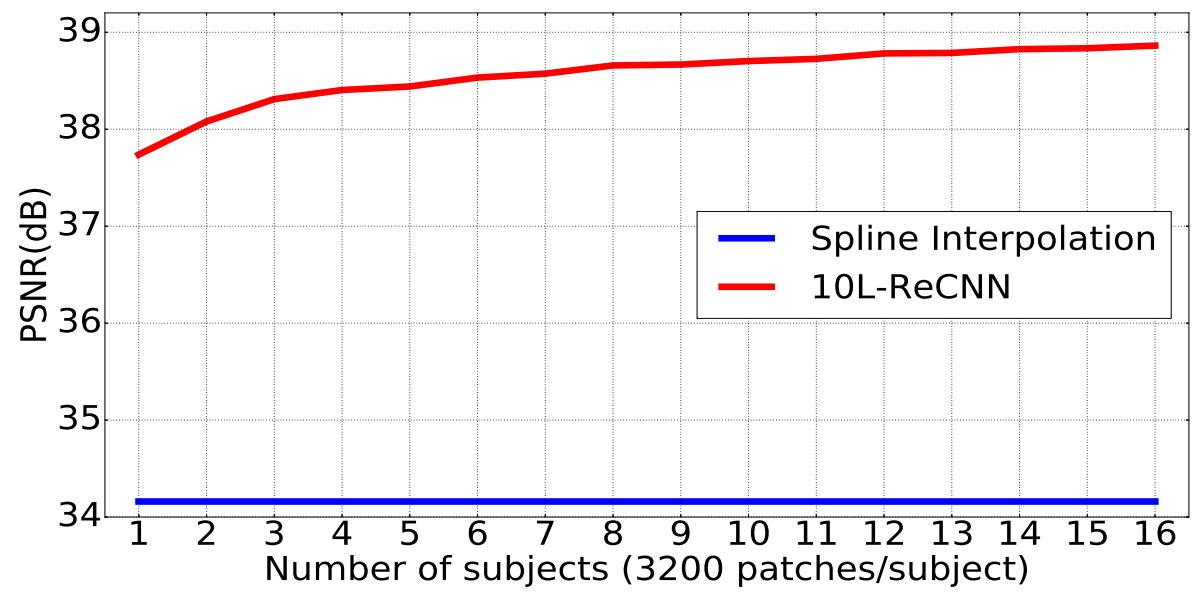

Figure 8: Number of subjects vs. performance (10-layer residual-learning networks with the same filter numbers $n=64$ and filter size $f=3$ over 20 training epochs using Adam optimization and tested with isotropic scale factor $\times 2$ using Kirby 21 for training and testing, 3200 patches per subject with size $25^{3}$ for training).

Following Kim et al. (2016a), we investigate how we may deal with multiple scales in a single network. It consists of creating a training dataset within which we consider LR and HR image pairs corresponding to different scaling factors. We test two cases: the first case where the learning dataset for combined scale factors $(\times 2, \times 3)$ has the same number as a single scale factor and the second one where we double the learning dataset for multiple scale factors. To avoid a convergence towards a local minimum of one of the scaling factors, we learn network parameters on randomly shuffled dataset.

Table 1 summarizes experimental results. First, when the training is achieved for the scaling from $(2 \times 2 \times 2)$ on a dataset of $(2 \times 2 \times 2)$ scale, it can be noticed that reconstruction performances decrease significantly when applied to other scaling factors (there is a drop from $39.01 \mathrm{~dB}$ to $33.43 \mathrm{~dB}$ when testing with $(3 \times 3 \times 3))$. Second, it can be noticed that when the training is performed on multiscale data within the same training samples, there is no significant performance change compared to training from a single-scale dataset. Third, a training dataset with double samples leads to a better performance. Moreover, training from multiple scaling factors leads to the estimation of a more versatile network. Overall, these results show that one single network can handle multiple arbitrary scaling factors.

\section{Multimodality-guided SR}

In clinical cases, it is frequent to acquire one isotropic HR image and LR images with different contrasts in order to reduce the acquisition time. Hence, a coplanar isotropic HR image might be considered as a complementary source of information to reconstruct HR MRI images from LR ones (Rousseau et al. 2010a). To address this multimodality-guided SR problem, we add a concatenation layer as the first layer of the network, as illustrated in Figure 9. This layer concatenates the ILR image and a registered HR reference along the 


\begin{tabular}{|c|c|c|c|c|}
\hline \multirow{2}{*}{ Test / Train } & \multicolumn{4}{|c|}{ Full-training } \\
\cline { 2 - 5 } & \multicolumn{3}{|c|}{ Same training samples } & Double samples \\
\cline { 2 - 5 } & $\times(2,2,2)$ & $\times(3,3,3)$ & $\times(2,2,2),(3,3,3)$ & $\times(2,2,2),(3,3,3)$ \\
\cline { 2 - 5 } & PSNR & PSNR & PSNR & PSNR \\
\hline$\times(2,2,2)$ & $\mathbf{3 9 . 0 1}$ & 35.25 & $\mathbf{3 7 . 3 5}$ & $\mathbf{3 8 . 8 0}$ \\
$\times(2,2,3)$ & 36.80 & 35.11 & 36.47 & 37.24 \\
$\times(2,2.5,2)$ & 37.71 & 35.41 & 36.91 & 37.93 \\
$\times(2,3,3)$ & 35.23 & 35.13 & 35.75 & 36.20 \\
$\times(2.5,2.5,2.5)$ & 35.47 & 35.52 & 36.09 & 36.63 \\
$\times(3,3,3)$ & 33.43 & $\mathbf{3 5 . 0 1}$ & $\mathbf{3 4 . 8 9}$ & $\mathbf{3 5 . 2 0}$ \\
\hline
\end{tabular}

Table 1: Experiments with multiple isotropic scaling factors with the 20-layers network using the training and testing images of Kirby 21. Bold numbers indicate that the tested scaling factor is present in the training dataset. We test two conditions of same training data and double training data.

channel axis. The registration step of HR reference ensures that the two input images share the same geometrical space.

We experimentally evaluate the relevance of the proposed multimodality-guided SR model according to the following setting. We investigate whether the complementary use of a Flair or a T2-weighted MRI image might be beneficial to improve the resolution of a LR T1-weighted MRI image. Concerning the Kirby 21 dataset, we apply an affine transform estimated using FSL (Jenkinson et al. 2012) to register images of a same subject into a common coordinate space. We assume here that the affine registration can compensate motion between two scans during the same acquisition session. This appears a fair assumption since here we are considering an organ that does not undergo significant deformation between two acquisitions. The registration step has been checked visually for all the images. Data of the NAMIC dataset are already in the same coordinate space so no registration step is required.

Figure 10 shows the results of the multimodality-guided SR compared to the monomodal SR for both Kirby dataset (a) and NAMIC datasets (b). It can be seen that multimodality driven approach can lead to improved reconstruction results. In these experiments, the overall upsampling result depends on the quality of the HR image used to drive the reconstruction process. Thus, adding high resolution information containing artifacts limits reconstruction performance. This is especially the case for the Kirby dataset. For instance, when considering $\mathrm{T} 2 \mathrm{w}$ images, no improvement is observed for Kirby dataset and an improvement greater than $1 d B$ is reported for NAMIC dataset. As the T2w image resolution is lower than T1w modality in Kirby dataset, these results may emphasize the requirement for actual HR information to expect significant gain w.r.t. the monomodal model. Figure 11 shows visually that edges in the residual image between the ground truth and the reconstruction by the multimodal approach are reduced significantly compared to interpolation and monomodal methods (e.g. the regions of lateral ventricles). This means that the multimodal approach resulting the reconstructions which are the most similar to the ground truth. These qualitative results highlight the fact that the proposed multimodal method provides a better performance than other compared methods. 


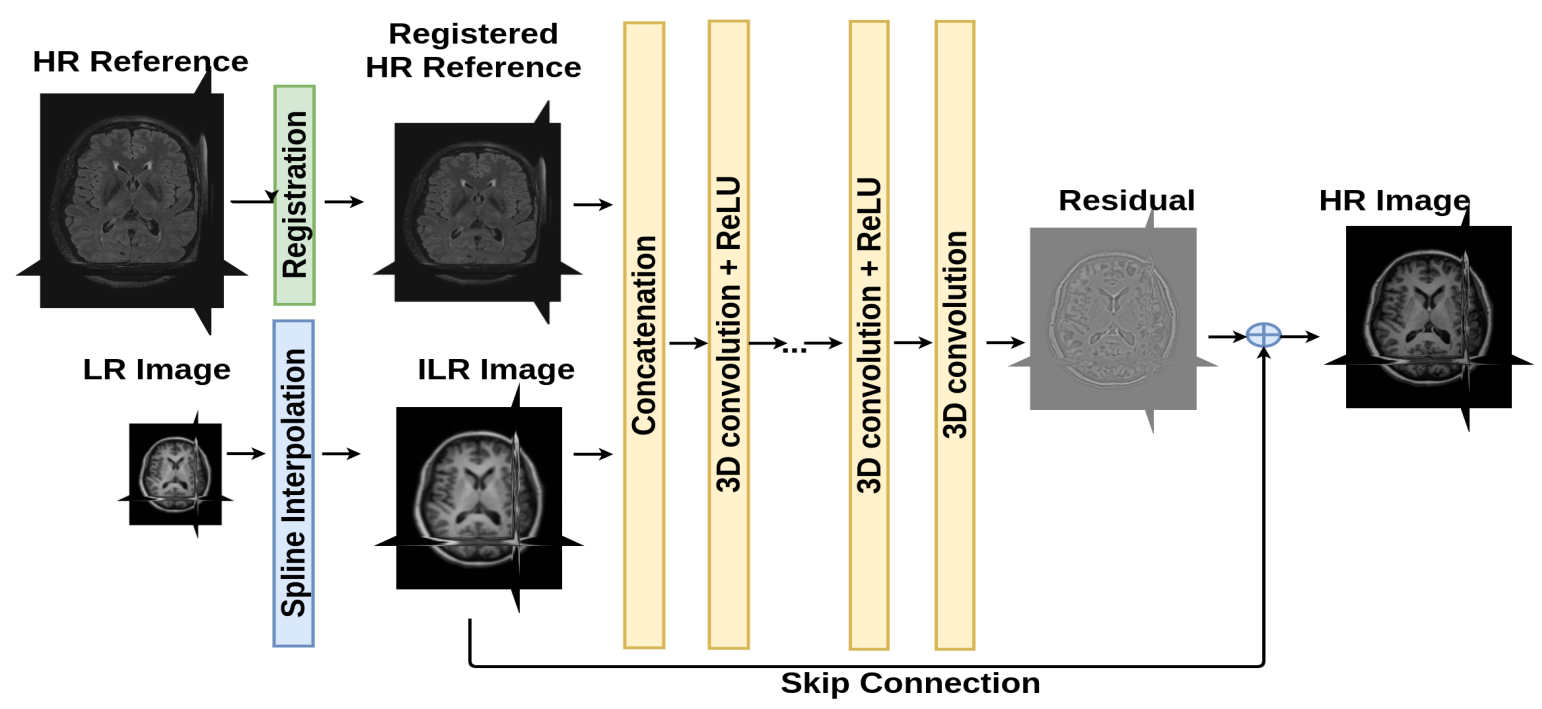

Figure 9: 3D deep neural network for multimodal brain MRI super-resolution using intermodality priors. Skip connection computes the residual between ILR image and HR image.

In addition, we explore the impact of the network depth augmentation with regard to the performance of multimodal SR approach. The experiments shown in Figure 12 indicate that the deeper structures do not lead to better results within the multimodal method.

\section{How Transferable are learnt Features?}

Training a CNN from scratch requires a substantial amount of training data and may take a long time. Moreover, to avoid overfitting, the training dataset has to reflect the appearance variability of the images to reconstruct. In the context of brain MRI, part of image variability comes from acquisition systems. Hence, we investigate the impact of such image variability onto SR performance by evaluating transfer learning skills among different datasets corresponding to the same imaging modality.

In order to characterize such generalization skills, we evaluate in which extent the selection of a given training dataset influences the reconstruction performance of the network. To this end, we train from scratch two 20L-ReCNN networks separately for a 10-image NAMIC T1-weighted dataset and a 10-image Kirby T1-weighted dataset, and we test the trained models for the remaining 10-image NAMIC and Kirby T1-weighted datasets. The considered case-study involves a scaling factor of $(2 \times 2 \times 2)$. For quantitative comparison, the PSNR and the structural similarity (SSIM) (the definition of SSIM can be found in Wang et al. 2004) are used to evaluate the performance of each model in Table 2. For benchmarking purposes, we also include a comparison with the following methods: cubic spline interpolation, low-rank total variation (LRTV) (Shi et al. 2015), SRCNN3D (Pham et al. 2017). The use of 20-layer CNN-based approaches for each training dataset can lead to improvements over spline interpolation, LRTV method and SRCNN3D (with respect to both PSNR and SSIM). Although, the gain is slightly lowered (e.g. PSNR: 0.55dB for testing Kirby and 0.74dB for NAMIC, SSIM: 0.003 for Kirby and 0.0019 for NAMIC) when 


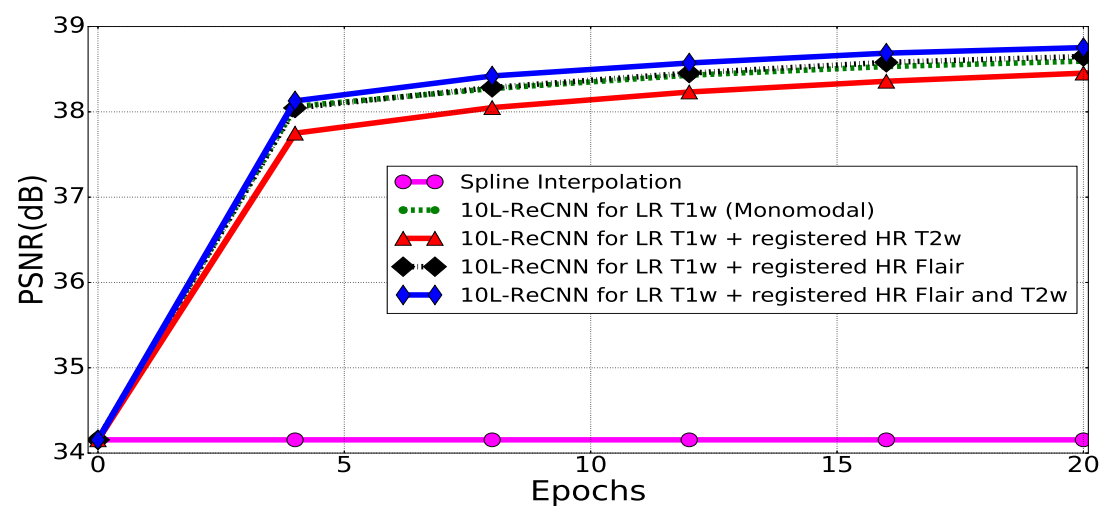

(a) Multimodal experiments using Kirby 21 dataset for training and testing.

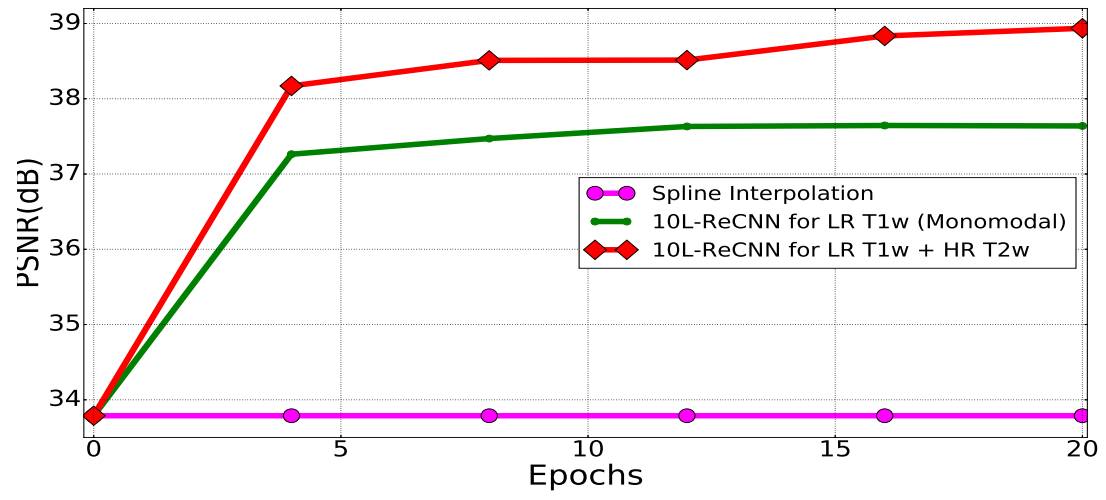

(b) Multimodal experiments using NAMIC dataset for training and testing.

Figure 10: Multimodality-guided SR experiments. The LR T1-weighted images are upscaled with isotropic scale factor $\times 2$ using respectively monomodal network (10L-ReCNN for LR T1w), HR T2w multimodal network, HR Flair multimodal network and both HR Flair and T2w multimodal images.

using different training and testing dataset (i.e. different resolution), our proposed networks obtain better results than compared methods.

For qualitative comparison, Figures 13 and 14 show the results of reconstructed 3D images obtained from all the compared techniques. The zoom version of the reconstructions 20L-ReCNN shows sharpen edges and a grayscale intensity which are closest to the ground truth. In addition, the HR reconstruction of the 20L-ReCNN model shows that its differences from the ground truth are less than other methods (i.e. the contours of the residual image of the 20L-ReCNN method are less visible than those of others). Hence, we can infer that our proposed method better preserves contours, geometrical structures and better recovers the image contrast compared with the other methods.

\section{Application of Super-Resolution on Clinical Neonatal Data}

In clinical routine, anisotropic LR MRI data are usually acquired in order to limit the acquisition time due to patient comfort such as infant brain MRI scans (Makropoulos et al., 2018), or in case of rapid emergency scans (Walter et al., 2003). These images usually have a high in-plane resolution and a low through-plane resolution. Anisotropic data acquisition 


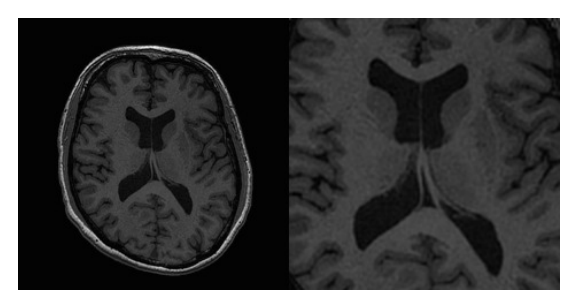

(a) Original HR T1-weighted

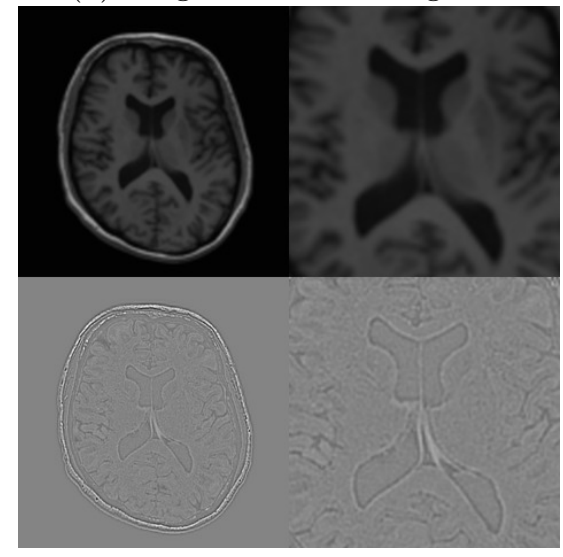

(d) Spline Interpolation

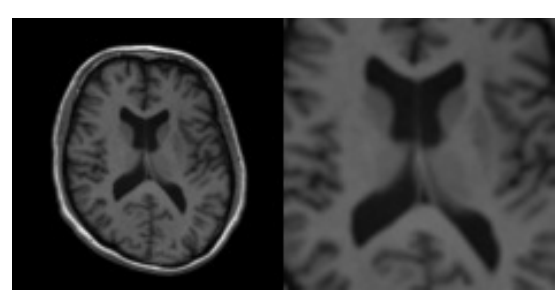

(b) LR T1-weighted image

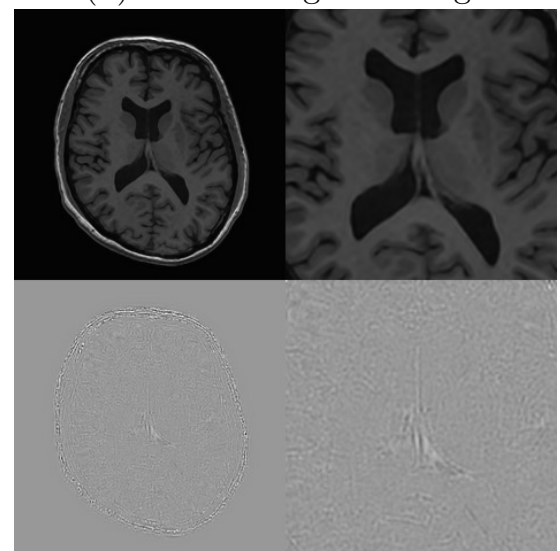

(e) Monomodal 10L-ReCNN

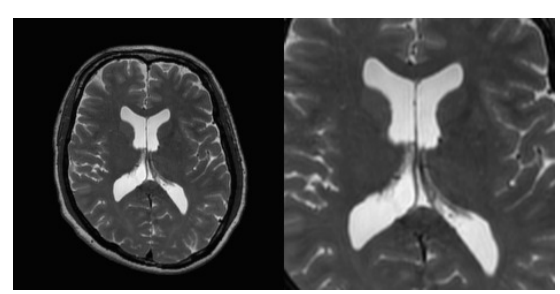

(c) HR T2-weighted reference

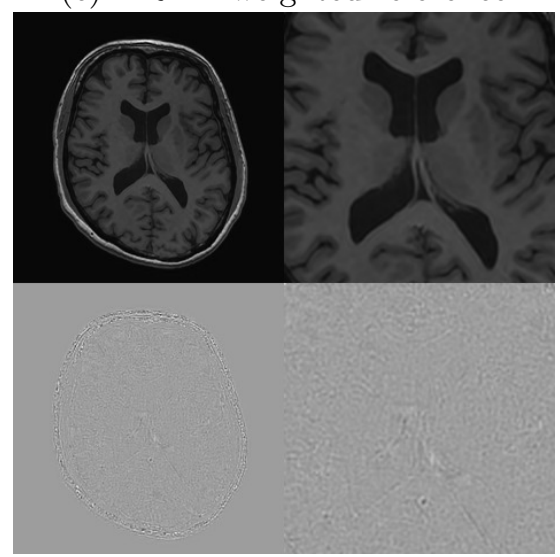

(f) Multimodal 10L-ReCNN

Figure 11: Illustration of the axial slices of monomodal and multimodal SR results (subject 01018, pathological case of testing set) with isotropic voxel upsampling using NAMIC. The LR T1-weighted image (b) with voxel size $2 \times 2 \times 2 \mathrm{~mm}^{3}$ is upsampled to size $1 \times 1 \times 1 \mathrm{~mm}^{3}$. The monomodal network 10L-ReCNN is applied directly the LR T1-weighted image (b), whereas the multimodal network 10L-ReCNN uses the HR T2-weighted reference (c) to upscale LR T1-weighted image (b). The results of the monomodal and multimodal networks are shown in (e) and (f), respectively. The different between ground truth image and reconstruction results are at the bottom. Their zoom version are at the right.

severely limits the 3D exploitation of MRI data. Interpolation is commonly used to upsampled these LR images to isotropic resolution. However, interpolated LR images may lead to partial volume artifacts that may affect segmentation (Ballester et al., 2002). In this section, we aim to use our single image SR method to enhance the resolution of such clinical data.

The idea is to apply our proposed convolutional neural networks-based SR method to transfer the rich information available from high-resolution experimental dataset to lowerquality image data. The procedure first uses CNNs to learn mappings between real HR images and their corresponding simulated LR images with the same resolution of real data. The LR data is generated by the observation model, which is decomposed into a linear downsampling operator after a space-invariant blurring model as a Gaussian kernel with the full-width-at-half-maximum (FWHM) equal to slice thickness (Greenspan, 2008). Once models are learnt, these mappings enhance the LR resolution of unseen low quality images.

In order to verify the applicability of our CNN-based methods, we have used two neonatal brain MRI dataset: the Developing Human Connectome Project (dHCP) (Hughes et al., 2017) and clinical neonatal MRI data acquired in the neonatology service of Reims Hospital (Multiphysics image-based AnalysIs for premature brAin development understanding 


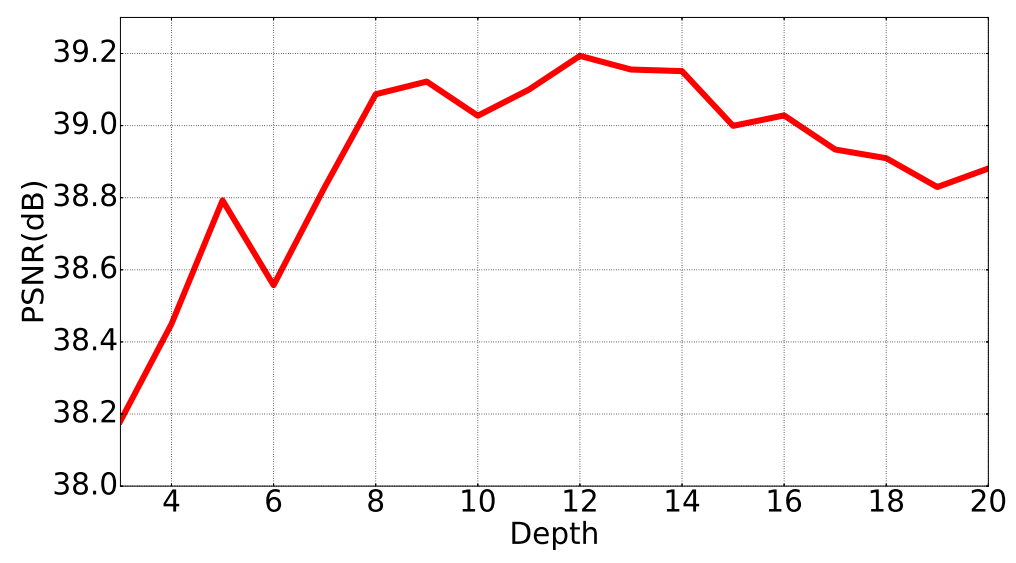

Figure 12: Depth vs. performance (multimodal SR using residual-learning networks with the same filter numbers $n=64$ and filter size $f=3$ over 20 training epochs using Adam optimization and tested with isotropic scale factor $\times 2$ using NAMIC for training and testing).

\begin{tabular}{|c|c|c|c|c|c|c|c|c|c|c|}
\hline \multirow{3}{*}{ Testing dataset } & \multirow{2}{*}{\multicolumn{2}{|c|}{ Spline Interpolation }} & \multirow{2}{*}{\multicolumn{2}{|c|}{ LRTV }} & \multirow{2}{*}{\multicolumn{2}{|c|}{$\begin{array}{c}\text { SRCNN3D } \\
\text { Kirby (10 images) }\end{array}$}} & \multicolumn{4}{|c|}{ 20L-ReCNN } \\
\hline & & & & & & & \multicolumn{2}{|c|}{ Kirby (10 images) } & \multicolumn{2}{|c|}{ NAMIC(10 images $)$} \\
\hline & PSNR & SSIM & PSNR & SSIM & PSNR & SSIM & PSNR & SSIM & PSNR & SSIM \\
\hline Kirby (5 images ) & 34.16 & 0.9402 & 35.08 & 0.9585 & 37.51 & 0.9735 & 38.93 & 0.9797 & 38.06 & 0.9767 \\
\hline Standard deviation & 1.90 & 0.0111 & 2.09 & 0.0083 & 1.97 & 0.0053 & 1.87 & 0.0044 & 1.83 & 0.0045 \\
\hline Gain & - & - & 0.92 & 0.0183 & 3.36 & 0.0333 & 4.77 & 0.0395 & 3.9 & 0.0365 \\
\hline NAMI & 33.78 & 9388 & 34.34 & 0.9549 & 36.72 & 0.9694 & 37.73 & 0.9762 & 38.28 & 0.9781 \\
\hline Standard deviation & 1.82 & 0.0071 & 1.79 & 0.0044 & 1.76 & 0.0035 & 1.81 & 0.0031 & 1.78 & 0.0029 \\
\hline Gain & - & - & 0.56 & 0.0161 & 2.94 & 0.0306 & 3.95 & 0.0374 & 4.5 & 0.0393 \\
\hline
\end{tabular}

Table 2: The results of PSNR/SSIM for isotropic scale factor $\times 2$ with the gain between compared methods and the method of spline interpolation. One network 20L-ReCNN trained with 10 images of Kirby and one trained with NAMIC

- MAIA dataset). The HR images are T2-weighted MRIs of the dHCP and provided by the Evelina Neonatal Imaging Centre, London, UK. Forty neonatal data were acquired on a 3T Achieva scanner with repetition (TR) of $12000 \mathrm{~ms}$ and echo times (TE) of $156 \mathrm{~ms}$, respectively. The size of voxels is $0.5 \times 0.5 \times 0.5 \mathrm{~mm}^{3}$. In-vivo neonatal LR images has a voxel size of about $0.4464 \times 0.4464 \times 3 \mathrm{~mm}^{3}$.

Figure 15 compares the qualitative results of HR reconstructions (spline interpolation, NMU (Manjón et al., 2010b) and our proposed networks) of a LR image from MAIA dataset. In this experiment, we do not have the ground truth of real LR data for calculating quantitative metrics. The comparison reveals that the CNNs-based methods recover shaper images and better defined boundaries than spline interpolation. For instance, the cerebrospinal fluid (CSF) of the cerebellum of the proposed method, in Figure 15, is more visible than those obtained with the compared methods. Our proposed technique reconstructs more curved cortex and more accurate ventricles. These results tend to confirm qualitatively the efficacy of our approach. 


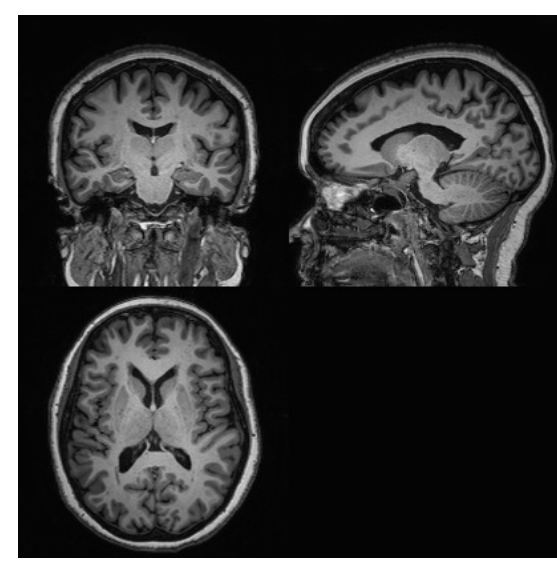

(a) Original HR

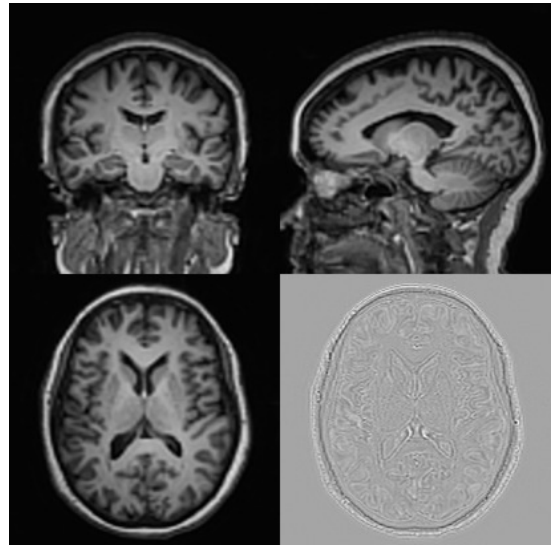

(d) LRTV

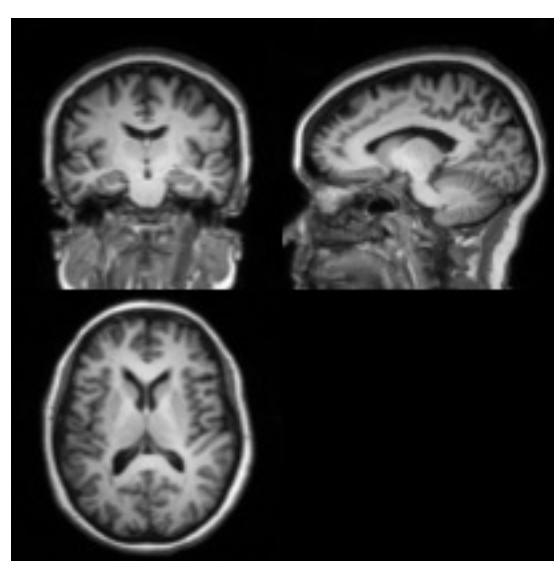

(b) LR image

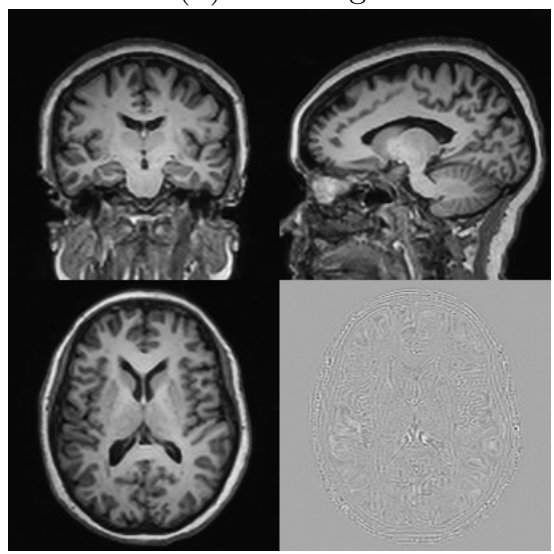

(e) SRCNN3D

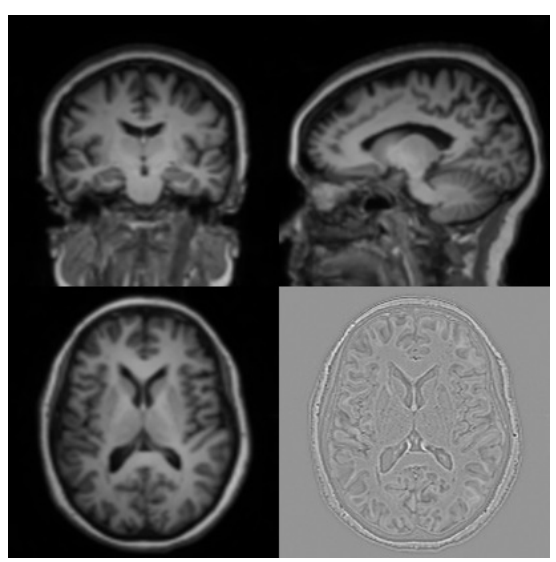

(c) Spline Interpolation

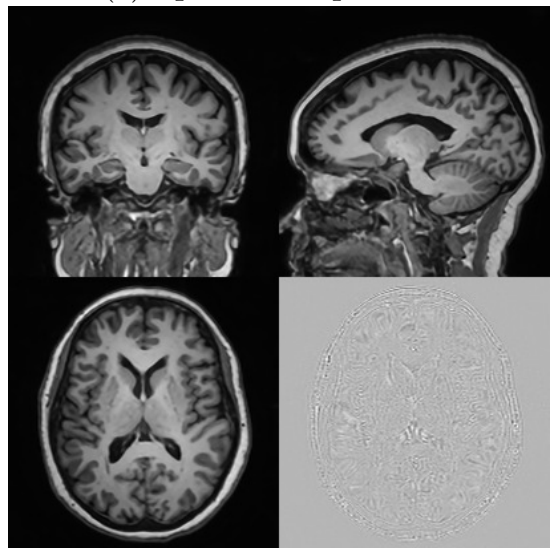

(f) 20L-ReCNN

Figure 13: Illustration of SR results (subject KKI2009-02-MPRAGE, non-pathological case, in testing set of dataset Kirby 21) with isotropic voxel upsampling. LR data (b) with voxel size $2 \times 2 \times 2.4 \mathrm{~mm}^{3}$ is upsampled to size $1 \times 1 \times 1.2 \mathrm{~mm}^{3}$. The difference between the ground truth image and the reconstruction results are in the right bottom corners. Both network SRCNN3D and network 20L-ReCNN are trained with the 10 last images of Kirby.

\section{Discussion}

This study investigates CNN-based models for 3D brain MR image SR. Based on a comprehensive experimental evaluation, we would like to draw the following conclusions and recommendations regarding the setup to be considered. We highlight that eight complementary factors may drive the reconstruction performance of CNN-based models. The combination of 1) appropriate optimization with 2) weight initialization and 3) residual learning is a key to exploit deeper networks with a faster and effective convergence. The choice of an appropriate optimization method can lead to a PSNR improvement of (at least) 1dB. In this study, it has appeared that Adam method (Kingma and Ba 2015) provides significantly better reconstruction results than other classical techniques such as SGD, and a faster convergence. Moreover, weights initialization is a very important step. Indeed, some approaches simply do not achieve convergence in the learning phase. This study has also 


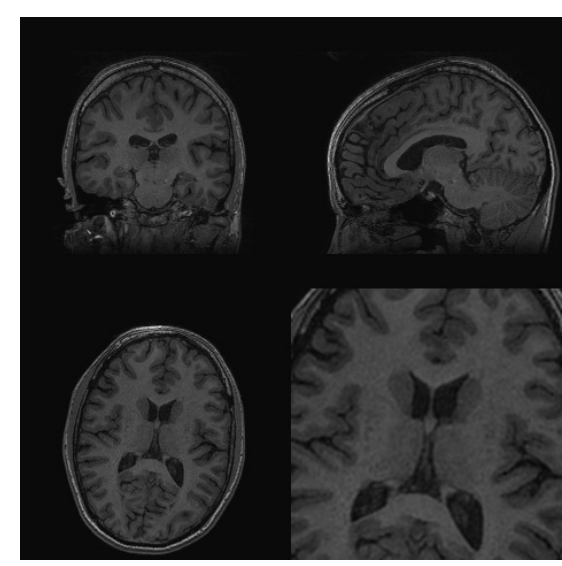

(a) Original HR

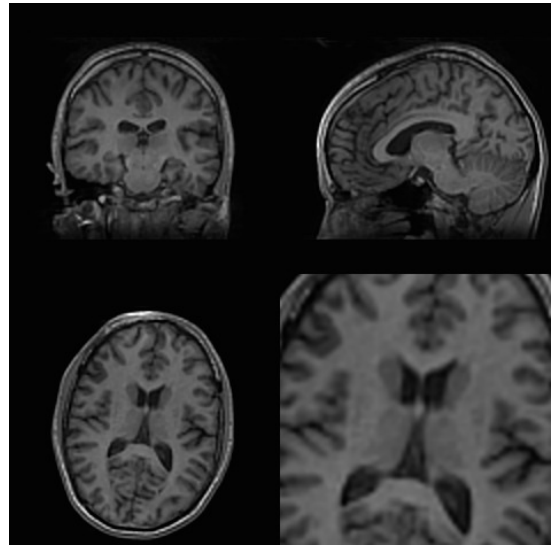

(d) Low-Rank Total Variation (LRTV)

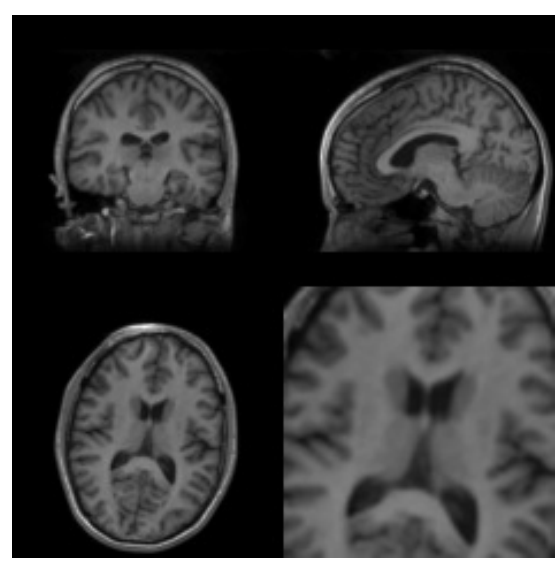

(b) LR image

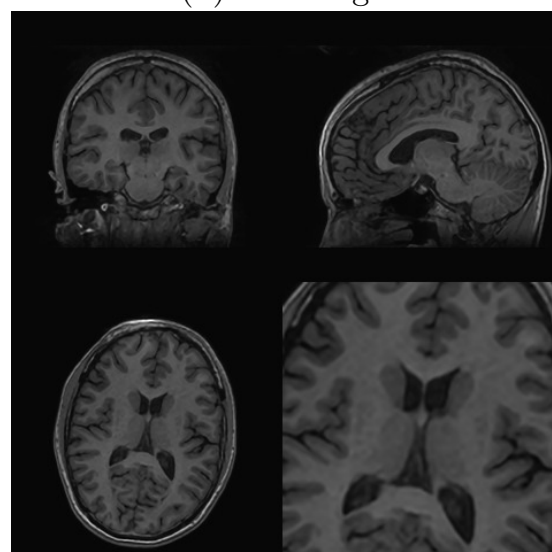

(e) 20L-ReCNN (trained with Kirby)

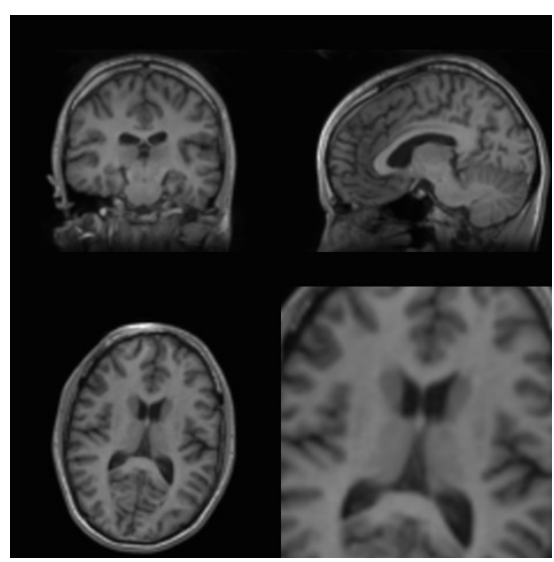

(c) Spline Interpolation

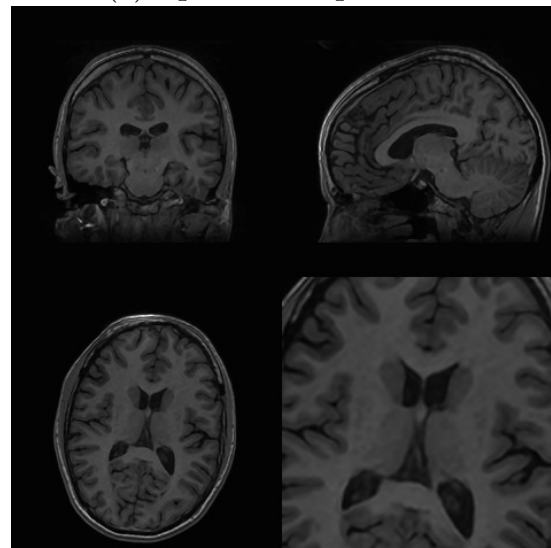

(f) 20L-ReCNN (trained with NAMIC)

Figure 14: Illustration of SR results (subject 01011-t1w, pathological case, in testing set of dataset NAMIC) with isotropic voxel upsampling. LR data (b) with voxel size $2 \times 2 \times 2 \mathrm{~mm}^{3}$ is upsampled to size $1 \times 1 \times 1 \mathrm{~mm}^{3}$. The zoom versions of the axial slices are in the right bottom corners.

shown that residual modeling for single image SR is a straightforward technique to improve the reconstruction performances $(+0.4 \mathrm{~dB})$ without requiring major changes in the network architecture. Appropriate weight initialization methods described by He et al. (2015); Glorot and Bengio (2010) allow us to build deeper residual-learning networks. From our point of view, these three aspects of SR algorithm are the first to require special attention for the implementation of a SR technique based on CNN.

Overall, we show that better performance can be achieved by learning 4) a deeper fully 3D convolution neural network, 5) exploring more filters and 6) increasing filter size. In addition, using 7) larger training patch size and 8) augmentation of training subject leads to increase the performance of the networks. The adjustment of these 5 elements provides a similar improvement (about 0.5dB). Although it seems natural to implement the deepest possible network, this parameter is not always the key to obtaining a better estimate of a highresolution image. Our study shows that, depending on the type of input data (monomodal or multimodal), network depth is not necessarily the main parameter leading to better 

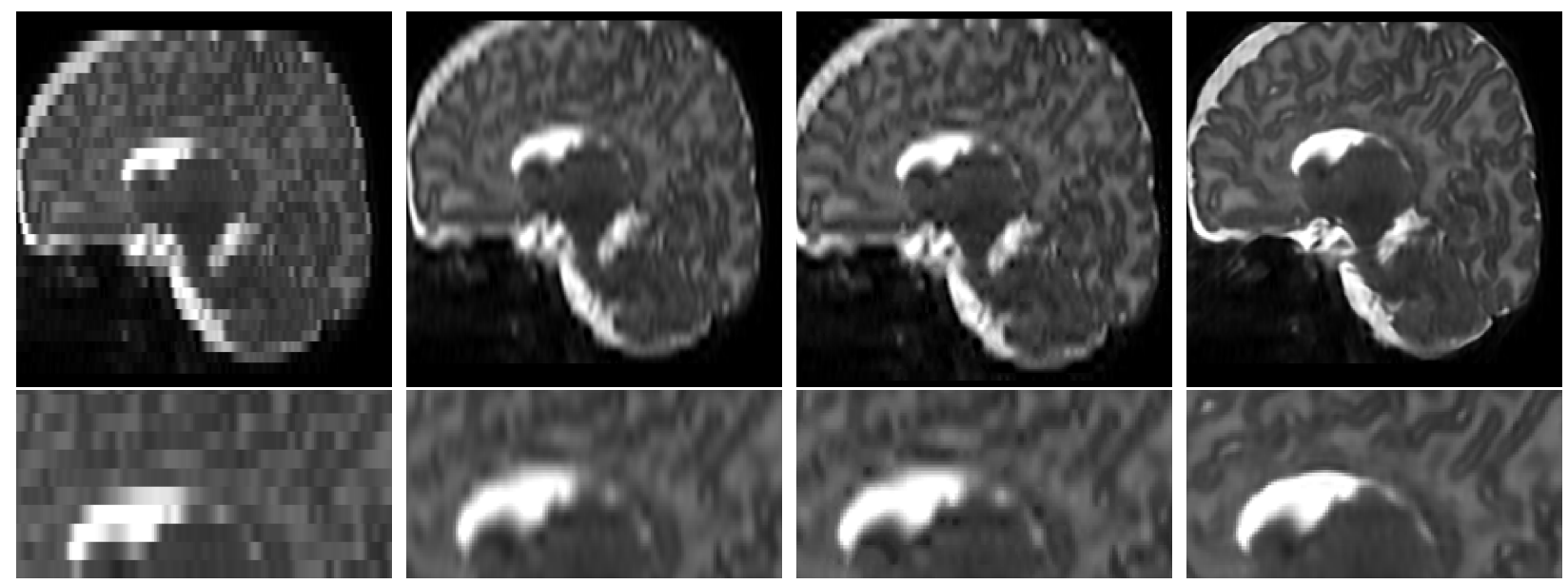

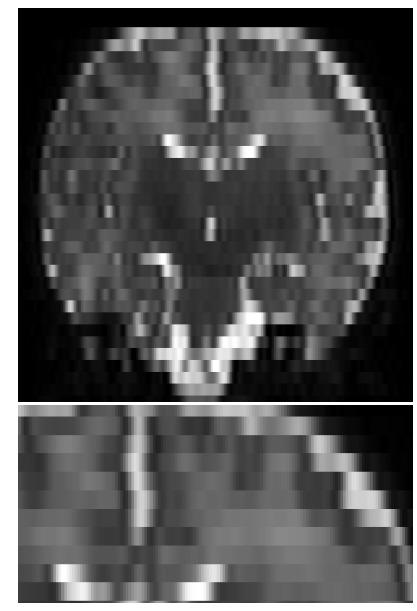

(a) Original LR image

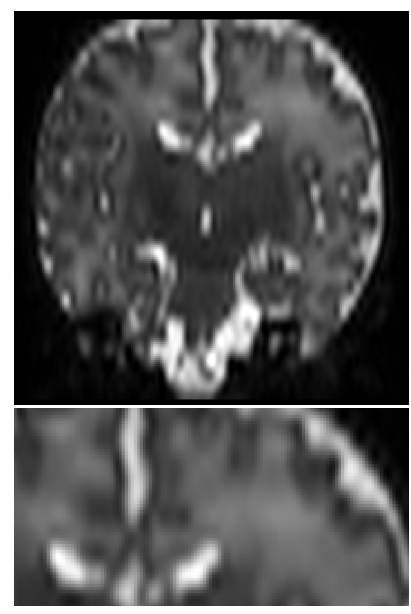

(b) Spline interpolation

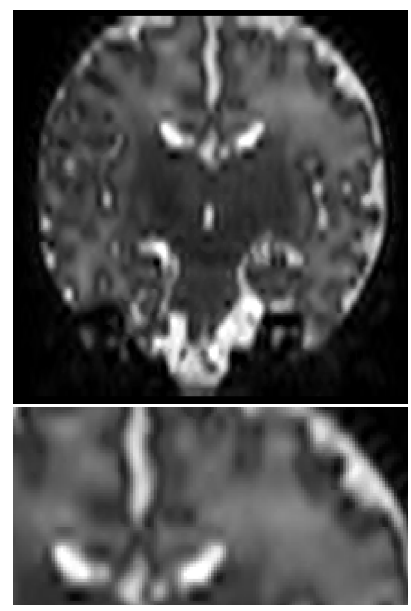

(c) NMU

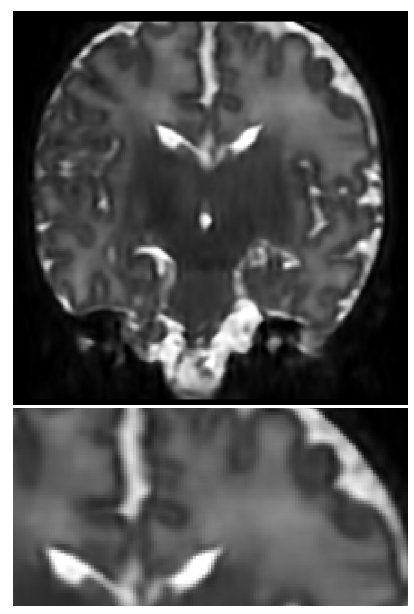

(d) Our proposed method

Figure 15: Illustration of SR results on a clinical data of MAIA dataset with isotropic voxel upsampling. Original data with voxel size of about $0.4464 \times 0.4464 \times 3$ is resampled to size $0.5 \times 0.5 \times 0.5 \mathrm{~mm}^{3}$. Networks are trained with the dHCP dataset. The first, second, third and last rows presents the sagittal slices, the zoom versions of the sagittal slices, the coronal slices and the zoom versions of the coronal slices, respectively.

image reconstruction. In addition, it is important to take into account the time of the learning phase as well as the maximum memory available in the GPU in order to choose the best architecture of the network. For instance, for the monomodal SR case based on the simulations of Kirby dataset, we suggest using 20-layer networks with 64 small filters with size of $3^{3}$ regarding 10 training subjects of size $25^{3}$ to achieve practicable results.

In CNN-based approaches, the upscaling operation can be performed by using transposed convolution (so-called fractionally strided convolutional) layers as proposed by Oktay et al. (2016); Dong et al. (2016b) or sub-pixel layers (Shi et al. 2016). However, the trained weights of these networks are totally applied for a specified scale factor. This is a limiting aspect of CNN-based SR for MR data since a fixed upscaling factor is not appropriate in this context. In this study, we have presented a multiscale CNN-based SR method for single 3D brain MRI that is capable of learning multiple scales by training multiple isotropic scale factors 


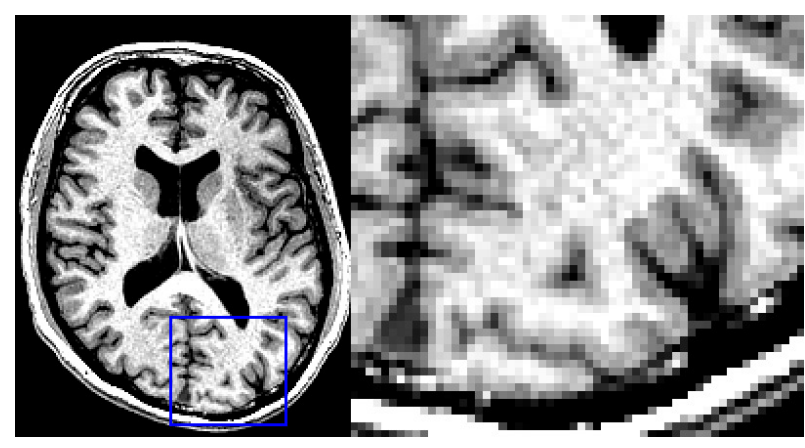

(a) Nearest-neighbor

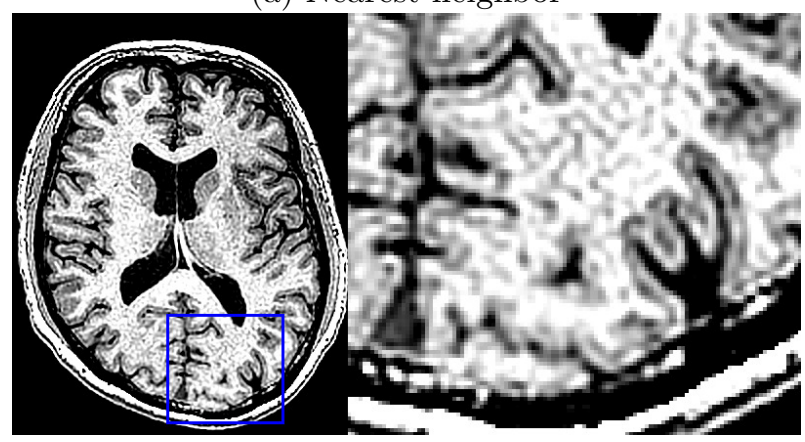

(c) LRTV

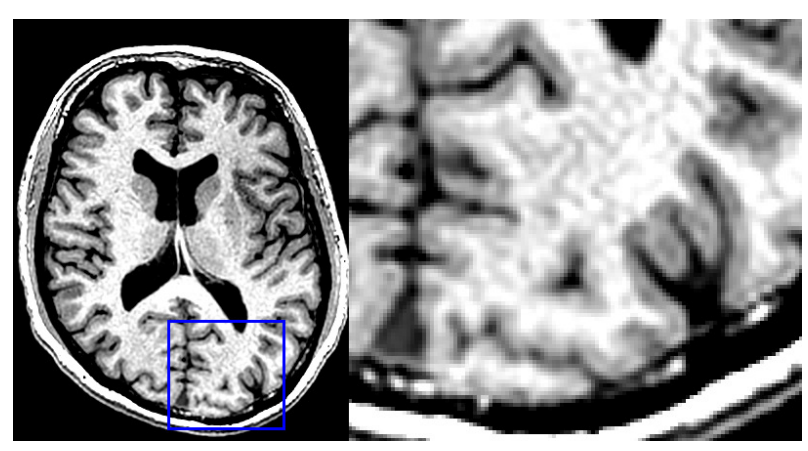

(b) Spline interpolation

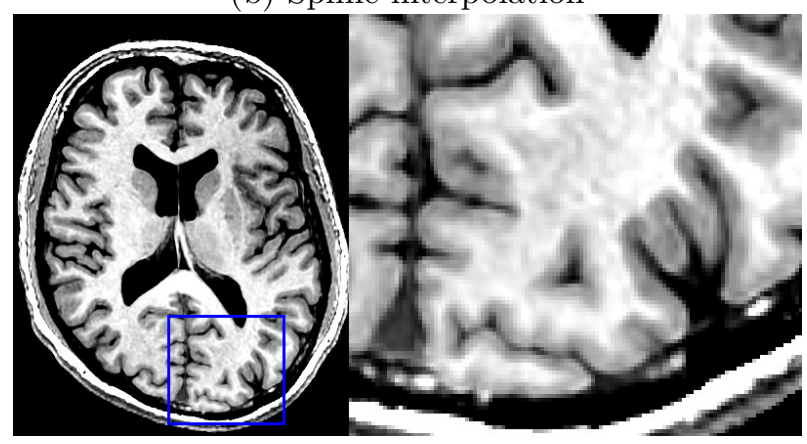

(d) 20L-ReCNN

Figure 16: Illustration of SR results (subject 01018-t1w in testing set of dataset NAMIC) with isotropic voxel upsampling. Original data with voxel size of $1 \times 1 \times 1 \mathrm{~mm}^{3}$ is upsampled to size $0.5 \times 0.5 \times 0.5 \mathrm{~mm}^{3}$. 20L-ReCNN is trained with the NAMIC dataset.

due to an independent upsampling technique such as spline interpolation. Handling multiple scales is related to multi-task learning. The lack of flexibility of learnt network architectures raises an open issue motivating further studies: how can we build a network that can deal with a set of observation models (i.e. multiple scales, arbitrary point spread functions, non uniform sampling, etc.)? For instance, when applying SR techniques in a realistic setting, the choice of the PSF is indeed a key element for SR methods and it depends on the type of MRI sequence. More specifically, the shape of the PSF depends on the trajectory in the k-space (Cartesian, radial, spiral). Making the network independent from the PSF model (i.e. doing blind SR) would be a major step for its use in routine protocol. Further research directions could focus on making more flexible CNN-based SR methods for greater use of these techniques in human brain mapping studies.

Evaluation of SR techniques is carried out on simulated LR images. However, one potential use of SR techniques would be to improve the resolution of isotropic data acquired in clinical routine. Figure 16 shows upsampling results on isotropic T1-weighted MR images (the resolution was increased from $1 \times 1 \times 1 \mathrm{~mm}^{3}$ to $0.5 \times 0.5 \times 0.5 \mathrm{~mm}^{3}$ ). In this experiment, the applied network has been trained to increase image resolution from $2 \times 2 \times 2 \mathrm{~mm}^{3}$ to $1 \times 1 \times 1 \mathrm{~mm}^{3}$. Although quantitative results cannot be computed, visual inspection of reconstructed upsampled images tend to show the potential of this SR method. Thus, features learnt at a lower scale ( $2 \mathrm{~mm}$ in this experiment) may be used to compute very 
high-resolution images that could be involved in fine studies of thin brain structures such as the cortex. Further work is required to investigate this aspect and more particularly the link with self-similarity based approaches.

In this article, we have proposed a multimodal method for brain MRI SR using CNNs where a HR reference image of the same subject can drive the reconstruction process of the LR image. By concatenating these HR and LR images, the reconstruction of the LR one can be enhanced by exploiting the multimodality feature of MR data. As shown in previous works (Rousseau et al. 2010a; Manjón et al. 2010a), the use of HR reference can lead to significant improvements of the reconstruction process. However, unlike the monomodal setup, a deeper network does not lead to better performance within the experiments on NAMIC dataset. Experiments from our study show that future work is needed to understand the relationship between network depth and the quality of HR image estimation.

Moreover, we have experimentally investigated the performances of CNN for generalizing on a different dataset (i.e. how a learnt network can be used in another context). More specifically, our study illustrates how knowledge learnt from one MR dataset is transferred to another one (different acquisition protocol and different scales). We have used Kirby and NAMIC datasets for this purpose. Although a slight decrease in performance can be observed, CNN-based approach can still achieve better performances than existing methods. These results tend to demonstrate the potential applications of CNN-based techniques for MRI SR. Further investigations are required to fully assess the possibilities of transfer learning in medical imaging context, and the contributions of fine-tuning technique (Tajbakhsh et al. 2016).

Finally, future research directions for CNN-based SR techniques could focus on other elements of the network architecture or the learning procedure. For instance, batch normalization (BN) step has been proposed by Ioffe and Szegedy (2015). The purpose of a BN layer is to normalize the data through the entire network, rather than just performing normalization once in the beginning. Although BN has been shown to improve classification accuracy and decrease training time (Ioffe and Szegedy 2015), we attempt to include BN layers into CNN for image SR but they do not lead to performance increase. Similar observations have been made in a recent SR challenge (Timofte et al. 2017). Moreover, whereas the classical MSE-based loss attempts to recover the smooth component, perceptual losses (Ledig et al. 2017; Johnson et al. 2016; Zhao et al. 2017) are proposed for natural image SR to better reconstruct fine details and edges. Thus, adding this type of layer (BN or residual block) or defining new loss functions may be beneficial for MRI SR and may provide new directions for research.

In this study, we have investigated the impact of adding data (about 3200 patches per added subject of Kirby dataset) on SR performances through PSNR computation. It appeared that using more subjects sightly improves the reconstruction results in this experimental setting. However, further work could focus on SR-specific data augmentation by rotation and flipping, which is usually used in many works (Kim et al. 2016a; Timofte et al. 2016), for improving algorithm generalization.

The evaluation on synthetic low-resolution images remains a limited approach to accurately quantify the performance of developed algorithms in real-world situations. However, 
acquiring low-resolution (LR) and high-resolution (HR) MRI data with the exact same contrast is very challenging. The development of such a database is beyond the scope of this work. There is currently no available dataset of pairs of real HR and LR images. This is the reason why all the works on SR perform quantitative analysis on synthetic data.

In order to demonstrate the potential of SR methods for enhancing the quality of clinical LR images, we have presented a practical application: image quality transfer from highresolution experimental dataset to real anisotropic low-resolution images. Our CNN-based SR method shows clear improvements over interpolation, which is the standard technique to enhance image quality from visualisation by a radiologist. SR method is therefore a highly relevant alternative to interpolation. Future work on the support of SR techniques for applications in brain segmentation could be investigated to evaluate the performance of these methods. Besides, the best way to evaluate the performances of SR methods is to gather datasets where pairs of real HR and LR images are available.

\section{Acknowledgement}

The research leading to these results has received funding from the ANR MAIA project, grant ANR-15-CE23-0009 of the French National Research Agency, INSERM, Institut Mines Télécom Atlantique (Chaire "Imagerie médicale en thérapie interventionnelle") and the American Memorial Hospital Foundation. We also gratefully acknowledge the support of NVIDIA Corporation with the donation of the Titan Xp GPU used for this research.

\section{References}

Ballester, M. A. G., Zisserman, A. P., Brady, M., 2002. Estimation of the partial volume effect in MRI. Medical Image Analysis 6 (4), 389-405.

Bengio, Y., Simard, P., Frasconi, P., 1994. Learning long-term dependencies with gradient descent is difficult. IIEEE Transactions on Neural Networks 5 (2), 157-166.

Chen, Y., Xie, Y., Zhou, Z., Shi, F., Christodoulou, A. G., Li, D., 2018. Brain MRI super resolution using 3D deep densely connected neural networks. In: 2018 IEEE 15th International Symposium on Biomedical Imaging (ISBI 2018). IEEE, pp. 739-742.

Dong, C., Loy, C. C., He, K., Tang, X., 2016a. Image super-resolution using deep convolutional networks. IEEE Transactions on Pattern Analysis and Machine Intelligence 38 (2), 295-307.

Dong, C., Loy, C. C., Tang, X., 2016b. Accelerating the super-resolution convolutional neural network. In: European Conference on Computer Vision. Springer, pp. 391-407.

Fogtmann, M., Seshamani, S., Kroenke, C., Cheng, X., Chapman, T., Wilm, J., Rousseau, F., Studholme, C., 2014. A unified approach to diffusion direction sensitive slice registration and 3D DTI reconstruction from moving fetal brain anatomy. IEEE Transactions on Medical Imaging 33 (2), 272-289.

Gholipour, A., Estroff, J. A., Warfield, S. K., 2010. Robust super-resolution volume reconstruction from slice acquisitions: application to fetal brain MRI. IEEE Transactions on Medical Imaging 29 (10), 1739-1758.

Glorot, X., Bengio, Y., 2010. Understanding the difficulty of training deep feedforward neural networks. In: Proceedings of the thirteenth International Conference on Artificial Intelligence and Statistics. pp. 249-256.

Greenspan, H., 2008. Super-resolution in medical imaging. The Computer Journal 52 (1), 43-63.

He, K., Zhang, X., Ren, S., Sun, J., 2015. Delving deep into rectifiers: Surpassing human-level performance on imagenet classification. In: Proceedings of the IEEE International Conference on Computer Vision. pp. 1026-1034. 
He, K., Zhang, X., Ren, S., Sun, J., 2016. Deep residual learning for image recognition. In: Proceedings of the IEEE Conference on Computer Vision and Pattern Recognition. pp. 770-778.

Huang, G., Liu, Z., Van Der Maaten, L., Weinberger, K. Q., 2017. Densely connected convolutional networks. In: Proceedings of the IEEE Conference on Computer Vision and Pattern Recognition. pp. 4700-4708.

Hughes, E., Grande, L. C., Murgasova, M., Hutter, J., Price, A., Gomes, A. S., Allsop, J., Steinweg, J., Tusor, N., Wurie, J., et al., 2017. The developing human connectome: announcing the first release of open access neonatal brain imaging. 23rd Annual Meeting of the Organization for Human Brain Mapping, $25-29$.

Ioffe, S., Szegedy, C., 2015. Batch normalization: Accelerating deep network training by reducing internal covariate shift. In: International Conference on Machine Learning. pp. 448-456.

Jain, S., Sima, D. M., Nezhad, F. S., Hangel, G., Bogner, W., Williams, S., Van Huffel, S., Maes, F., Smeets, D., 2017. Patch-based super-resolution of MR spectroscopic images: Application to multiple sclerosis. Frontiers in neuroscience 11.

Jenkinson, M., Beckmann, C. F., Behrens, T. E., Woolrich, M. W., Smith, S. M., 2012. Fsl. Neuroimage $62(2), 782-790$.

Jia, Y., Gholipour, A., He, Z., Warfield, S. K., 2017. A new sparse representation framework for reconstruction of an isotropic high spatial resolution MR volume from orthogonal anisotropic resolution scans. IEEE Transactions on Medical Imaging 36 (5), 1182-1193.

Jia, Y., Shelhamer, E., Donahue, J., Karayev, S., Long, J., Girshick, R., Guadarrama, S., Darrell, T., 2014. Caffe: Convolutional architecture for fast feature embedding. In: Proceedings of the 22nd ACM International Conference on Multimedia. ACM, pp. 675-678.

Jog, A., Carass, A., Prince, J. L., 2016. Self super-resolution for magnetic resonance images. In: International Conference on Medical Image Computing and Computer-Assisted Intervention. Springer, pp. 553-560.

Johnson, J., Alahi, A., Fei-Fei, L., 2016. Perceptual losses for real-time style transfer and super-resolution. In: European Conference on Computer Vision. Springer, pp. 694-711.

Kainz, B., Steinberger, M., Wein, W., Kuklisova-Murgasova, M., Malamateniou, C., Keraudren, K., TorsneyWeir, T., Rutherford, M., Aljabar, P., Hajnal, J. V., et al., 2015. Fast volume reconstruction from motion corrupted stacks of 2D slices. IEEE Transactions on Medical Imaging 34 (9), 1901-1913.

Kamnitsas, K., Ledig, C., Newcombe, V. F., Simpson, J. P., Kane, A. D., Menon, D. K., Rueckert, D., Glocker, B., 2017. Efficient multi-scale 3D CNN with fully connected CRF for accurate brain lesion segmentation. Medical Image Analysis 36, 61-78.

Kim, J., Lee, J. K., Lee, K. M., 2016a. Accurate image super-resolution using very deep convolutional networks. In: in Proceedings of IEEE Conference on Computer Vision and Pattern Recognition.

Kim, J., Lee, J. K., Lee, K. M., 2016b. Deeply-recursive convolutional network for image super-resolution. In: in Proceedings of IEEE Conference on Computer Vision and Pattern Recognition.

Kingma, D., Ba, J., 2015. Adam: A method for stochastic optimization. In: International Conference on Learning Representations.

Krizhevsky, A., Sutskever, I., Hinton, G. E., 2012. Imagenet classification with deep convolutional neural networks. In: Advances in Neural Information Processing Systems. pp. 1097-1105.

Landman, B. A., Huang, A. J., Gifford, A., Vikram, D. S., Lim, I. A. L., Farrell, J. A., Bogovic, J. A., Hua, J., Chen, M., Jarso, S., et al., 2011. Multi-parametric neuroimaging reproducibility: a 3T resource study. Neuroimage 54 (4), 2854-2866.

LeCun, Y., Bottou, L., Bengio, Y., Haffner, P., 1998. Gradient-based learning applied to document recognition. Proceedings of the IEEE 86 (11), 2278-2324.

Ledig, C., Theis, L., Huszár, F., Caballero, J., Cunningham, A., Acosta, A., Aitken, A., Tejani, A., Totz, J., Wang, Z., et al., 2017. Photo-realistic single image super-resolution using a generative adversarial network. In: in Proceedings of IEEE Conference on Computer Vision and Pattern Recognition.

Lim, B., Son, S., Kim, H., Nah, S., Lee, K. M., 2017. Enhanced deep residual networks for single image superresolution. In: The IEEE Conference on Computer Vision and Pattern Recognition (CVPR) Workshops. Vol. 1. p. 3.

Makropoulos, A., Robinson, E. C., Schuh, A., Wright, R., Fitzgibbon, S., Bozek, J., Counsell, S. J., Steinweg, 
J., Vecchiato, K., Passerat-Palmbach, J., et al., 2018. The developing human connectome project: A minimal processing pipeline for neonatal cortical surface reconstruction. Neuroimage 173, 88-112.

Manjón, J. V., Coupé, P., Buades, A., Collins, D. L., Robles, M., 2010a. MRI superresolution using selfsimilarity and image priors. Journal of Biomedical Imaging 2010, 17.

Manjón, J. V., Coupé, P., Buades, A., Fonov, V., Collins, D. L., Robles, M., 2010b. Non-local MRI upsampling. Medical Image Analysis 14 (6), 784-792.

Milanfar, P., 2010. Super-Resolution Imaging. CRC press.

Nesterov, Y., 1983. A method of solving a convex programming problem with convergence rate $o\left(1 / k^{2}\right)$. In: Soviet Mathematics Doklady. Vol. 27. pp. 372-376.

Oktay, O., Bai, W., Lee, M., Guerrero, R., Kamnitsas, K., Caballero, J., de Marvao, A., Cook, S., O'Regan, D., Rueckert, D., 2016. Multi-input cardiac image super-resolution using convolutional neural networks. In: International Conference on Medical Image Computing and Computer-Assisted Intervention. Springer, pp. $246-254$.

Pascanu, R., Mikolov, T., Bengio, Y., 2013. On the difficulty of training recurrent neural networks. ICML (3) 28, 1310-1318.

Pham, C.-H., Ducournau, A., Fablet, R., Rousseau, F., 2017. Brain MRI super-resolution using deep 3D convolutional networks. In: Biomedical Imaging (ISBI 2017), 2017 IEEE 14th International Symposium on. IEEE, pp. 197-200.

Pham, C.-H., Tor-Díez, C., Meunier, H., Bednarek, N., Fablet, R., Passat, N., Rousseau, F., 2019. Simultaneous super-resolution and segmentation using a generative adversarial network: Application to neonatal brain MRI. In: 2019 IEEE 16th International Symposium on Biomedical Imaging (ISBI 2019). IEEE.

Poot, D. H., Jeurissen, B., Bastiaensen, Y., Veraart, J., Van Hecke, W., Parizel, P. M., Sijbers, J., 2013. Super-resolution for multislice diffusion tensor imaging. Magnetic Resonance in Medicine 69 (1), 103-113.

Ramos-Llordén, G., Arnold, J., Van Steenkiste, G., Jeurissen, B., Vanhevel, F., Van Audekerke, J., Verhoye, M., Sijbers, J., 2017. A unified maximum likelihood framework for simultaneous motion and $t_{-}\{1\}$ estimation in quantitative $\mathrm{mr} t_{-}\{1\}$ mapping. IEEE Transactions on Medical Imaging 36 (2), 433-446.

Rousseau, F., Initiative, A. D. N., et al., 2010a. A non-local approach for image super-resolution using intermodality priors. Medical Image Analysis 14 (4), 594-605.

Rousseau, F., Kim, K., Studholme, C., Koob, M., Dietemann, J.-L., 2010b. On super-resolution for fetal brain MRI. In: International Conference on Medical Image Computing and Computer-Assisted Intervention. Springer, pp. 355-362.

Rueda, A., Malpica, N., Romero, E., 2013. Single-image super-resolution of brain MR images using overcomplete dictionaries. Medical Image Analysis 17 (1), 113-132.

Scherrer, B., Gholipour, A., Warfield, S. K., 2012. Super-resolution reconstruction to increase the spatial resolution of diffusion weighted images from orthogonal anisotropic acquisitions. Medical Image Analysis 16 (7), 1465-1476.

Shi, F., Cheng, J., Wang, L., Yap, P., Shen, D., 2015. LRTV: MR image super-resolution with low-rank and total variation regularizations. IEEE Transactions on Medical Imaging 34 (12), 2459-2466.

Shi, W., Caballero, J., Huszar, F., Totz, J., Aitken, A. P., Bishop, R., Rueckert, D., Wang, Z., 2016. Realtime single image and video super-resolution using an efficient sub-pixel convolutional neural network. In: Proceedings of the IEEE Conference on Computer Vision and Pattern Recognition. pp. 1874-1883.

Simonyan, K., Zisserman, A., 2014. Very deep convolutional networks for large-scale image recognition. In: International Conference on Learning Representations.

Steenkiste, G., Jeurissen, B., Veraart, J., Den Dekker, A. J., Parizel, P. M., Poot, D. H., Sijbers, J., 2016. Super-resolution reconstruction of diffusion parameters from diffusion-weighted images with different slice orientations. Magnetic Resonance in Medicine 75 (1), 181-195.

Tajbakhsh, N., Shin, J. Y., Gurudu, S. R., Hurst, R. T., Kendall, C. B., Gotway, M. B., Liang, J., 2016. Convolutional neural networks for medical image analysis: full training or fine tuning? IEEE Transactions on Medical Imaging 35 (5), 1299-1312.

Tieleman, T., Hinton, G., 2012. Lecture 6.5-rmsprop: Divide the gradient by a running average of its recent magnitude. COURSERA: Neural networks for machine learning 4 (2), 26-31. 
Timofte, R., Agustsson, E., Van Gool, L., Yang, M.-H., Zhang, L., et al., 2017. NTIRE 2017 challenge on single image super-resolution: Methods and results. In: The IEEE Conference on Computer Vision and Pattern Recognition (CVPR) Workshops.

Timofte, R., De Smet, V., Van Gool, L., 2013. Anchored neighborhood regression for fast example-based super-resolution. In: Computer Vision (ICCV), 2013 IEEE International Conference on. IEEE, pp. 19201927.

Timofte, R., De Smet, V., Van Gool, L., 2014. A+: Adjusted anchored neighborhood regression for fast super-resolution. In: Computer Vision-ACCV 2014. Springer, pp. 111-126.

Timofte, R., Rothe, R., Van Gool, L., 2016. Seven ways to improve example-based single image super resolution. In: Proceedings of the IEEE Conference on Computer Vision and Pattern Recognition. pp. $1865-1873$.

Tor-Díez, C., Pham, C.-H., Meunier, H., Faisan, S., Bloch, I., Bednarek, N., Passat, N., Rousseau, F., 2019. Evaluation of cortical segmentation pipelines on clinical neonatal MRI data. In: 41st International Engineering in Medicine and Biology Conference (EMBC 2019).

Van Steenkiste, G., Poot, D. H., Jeurissen, B., Den Dekker, A. J., Vanhevel, F., Parizel, P. M., Sijbers, J., 2017. Super-resolution T1 estimation: Quantitative high resolution T1 mapping from a set of low resolution T1-weighted images with different slice orientations. Magnetic Resonance in Medicine 77 (5), $1818-1830$.

Walter, C., Kruessell, M., Gindele, A., Brochhagen, H., Gossmann, A., Landwehr, P., 2003. Imaging of renal lesions: evaluation of fast MRI and helical CT. The British Journal of Radiology 76 (910), 696-703.

Wang, Z., Bovik, A. C., Sheikh, H. R., Simoncelli, E. P., 2004. Image quality assessment: from error visibility to structural similarity. IEEE Transactions on Image Processing 13 (4), 600-612.

Zeyde, R., Elad, M., Protter, M., 2012. On single image scale-up using sparse-representations. In: Curves and Surfaces. Springer, pp. 711-730.

Zhao, C., Carass, A., Dewey, B. E., Prince, J. L., 2018. Self super-resolution for magnetic resonance images using deep networks. In: 2018 IEEE 15th International Symposium on Biomedical Imaging (ISBI 2018). IEEE, pp. 365-368.

Zhao, H., Gallo, O., Frosio, I., Kautz, J., 2017. Loss functions for neural networks for image processing. IEEE Transactions on Computational Imaging 2017. 